\title{
"Magische Momente" im Klassenraum? Die Perspektive von Lehrkrätten und Lernenden zum Einsatz von Spielfilmen in universitären und außeruniversitären Deutschprogrammen in Malaysia
}

["Magical Moments" in the Classroom? The Perspective of Teachers and Learners on the Use of Feature Films in (university-taught and non-university) German Language Programs in Malaysia]

http://dx.doi.org/10.11606/1982-8837213587

\author{
Torsten Schaar ${ }^{1}$ \\ Shi Wen Chans ${ }^{2}$ \\ Annika Gilgen ${ }^{3}$
}

\begin{abstract}
Feature films in foreign-language education are a modern, efficient, motivating, stimulating and engaging medium for conveying and experiencing authentic language in real communication situations, for teaching target-oriented, country-specific cultural information as well as for developing intercultural awareness and communication skills. The paper examines the extent to which the use of feature film is actually implemented in L3 German classrooms in Malaysia. Based on two empirical studies conducted via standardized questionnaires and interviews with 43 teachers and 168 learners of German as a Foreign Language, the aim was to examine the importance of film material as a didactic instrument in German language programs and to analyze the motifs and didactic goals for implementing films. Students were interviewed about their media attitudes, their film viewing habits and genre preferences as well as about their perception of aspects of German culture in feature films. Although Malaysian learners of the German language show a great interest in Germany, its people, language, society and culture, teachers - for a variety of reasons but foremost caused by a lack of knowledge about the adequate implementation of feature films - have provided only few "magical moments" in L3 German classrooms thus far.
\end{abstract}

Keywords: Learning German in Malaysia, feature films in learning German as a Foreign Language in Malaysia; motives for implementing feature films in the German as a Foreign Language classroom, empirical studies, electronic survey, genre preferences

\footnotetext{
1 University Putra Malaysia, Department of Foreign Languages, German Unit, Serdang, Selangor, 43400, Malaysia. E-mail: TorstenSchaar@web.de.

${ }^{2}$ University Putra Malaysia, UPM, Serdang, Selangor, 43400, Malaysia. E-mail: xantthe @ gmail.com.

${ }^{3}$ University Putra Malaysia, UPM, Serdang, Selangor, 43400, Malaysia. E-mail: gilgenannika@ gmx.de.
}

\section{(cc) BY-NC}


Schaar, T.; Chang, S.W.; Gilgen, A. - "Magische Momente" im Klassenraum?

Zusammenfassung: Spielfilme als dominante Ausdrucksform kultureller Identität sind ein modernes, motivierendes, emotional ansprechendes und effizientes (ergänzendes) Lehr- und Lernmedium im Sprachunterricht, dass die Lernenden in ,realistisch wirkende Kommunikation“ und aktuelle Landeskunde einführt sowie interkulturelle kommunikative Kompetenz entwickelt. Das Interesse der Deutschlernenden an Spielfilmen ist groß und vor allem auf das Erleben authentischer Sprache in realen Situationen, auf den Alltag und die zwischenmenschlichen Beziehungen in deutschsprachigen Ländern ausgerichtet. In der Mehrzahl der außeruniversitären DaF-Programme in Malaysia ist das Medium Film trotz aller theoretischen Einsichten seitens der Lehrenden noch immer unterrepräsentiert und beschränkt sich zumeist auf reine Unterhaltung bzw. auf die Vermittlung von landeskundlichen Aspekten. Die Ursachen liegen in der fehlenden Aus- und Fortbildung im Bereich Filmarbeit im Fremdsprachenunterricht und der sich daraus ergebenden Unsicherheit der Lehrkräfte bei der zielgerichteten Implementierung filmischer Textsorten im Unterricht. Hinzu kommen ein stark prüfungszentrierter, traditionell grammatikorientierter Unterricht sowie Zeitmangel, aber auch das Desinteresse vieler Lehrender an der aufwändigen didaktisch-methodischen Vorentlastung und Nachbereitung einer Filmvorführung. Lediglich in den BA-German Programmen werden Filme in verschiedenen Kursen zur Vermittlung von Sprache, Landeskunde, Geschichte, Literatur, Kunst und zum fremd- und eigenkulturellen Vergleich eingesetzt. Am German-Malaysian-Institute dienen deutsche Spielfilme vor allem der interkulturellen Vorbereitung künftiger Studierender ingenieurswissenschaftlicher Programme an deutschen Fachhochschulen.

Stichwörter: Deutschlernen in Malaysia, deutsche Spielfilme im Unterricht Deutsch als Fremdsprache, Motive für die Implementierung von Spielfilmen im DaF-Unterricht, elektronische Umfragen, Genrepräferenzen

\section{Einführende Bemerkungen - Deutsch in Malaysia}

Die Sektion „Film und Video“ der XIV. Internationalen Tagung der Deutschlehrerinnen und Deutschlehrer (IDT 2009) in Jena/Weimar konstatierte eine insgesamt positive Entwicklung hinsichtlich des Stellenwertes des Mediums Film im Deutsch als Fremdsprache/Deutsch als Zweitsprache (DaF/DaZ)-Unterricht in den letzten Jahren. Es wurde jedoch auch kritisch angemerkt, dass Filme ,noch kein fixer Bestandteil“ des täglichen DaF/DaZUnterrichtsgeschehens sind, dass sie eher als „Belohnung und ,Stundenfüller“ zu besonderen Anlässen“ eingesetzt werden. Als wesentliche Ursache dafür wird die fehlende Medienkompetenz der Deutschlehrenden benannt (WELKE; FAISTAUER 2010: 7-8). Diese Aussagen lassen sich auch auf die Arbeit mit dem Medium Film in den Deutschprogrammen in Malaysia übertragen. Kaum ein Medium prägt und dominiert die Freizeitgestaltung und Lebenswelt junger Menschen in Malaysia so stark wie der Film (SCHAAR; LAPASAU; NG 2013), dennoch erfährt insbesondere der Spielfilm im Fremdsprachenunterricht eine eher geringe Beachtung.

Der vorliegende Artikel ist in vier Teile gegliedert. Einführend wird die Situation der deutschen Sprache in Malaysia vorgestellt, anschließend werden der Stellenwert und die

Pandaemonium, São Paulo, v. 21, n. 35, set.-dez. 2018, p. 87-120 
Schaar, T.; Chang, S.W.; Gilgen, A. - "Magische Momente" im Klassenraum?

Gründe für eine gezielte Implementierung von filmischen Textsorten, insbesondere von Spielfilmen im DaF/DaZ-Unterricht diskutiert. Der Schwerpunkt liegt auf der Vorstellung der Ergebnisse zweier elektronischer Umfragen unter Deutschlehrenden und -lernenden zum Einsatz von Medien im Allgemeinen und von deutschen Spielfilmen im Besonderen in universitären und außeruniversitären Deutschprogrammen in Malaysia.

Das Studium von Sprachen spielt eine wichtige Rolle im Bildungssystem Malaysias, da Sprachkenntnisse als ein entscheidender Faktor, nicht nur für die Identitätsbildung der Nation, sondern auch für die erfolgreiche Entwicklung der Wirtschaft und Wissenschaft des Landes identifiziert wurden. Um junge Malaysierinnen und Malaysier zu befähigen, auf globaler Ebene kommunizieren und somit konkurrieren zu können, richteten öffentliche und private Hochschulen in ganz Malaysia Sprachabteilungen für Bahasa Malaysia, für Englisch sowie für europäische und asiatische Sprachen wie Arabisch, Burmesisch, Französisch, Italienisch, Japanisch, Koreanisch, Türkisch, Russisch, Spanisch, Thai und auch für Deutsch ein (MINISTRY OF Higher EDUCATION 2010).

Seit der Einführung des Faches Deutsch als Fremdsprache an 8 Residential Schools (Internatsschulen) im Jahre 1995 ist das Interesse am Deutschlernen in Malaysia stetig gewachsen. Die wesentliche Motivation für das Erlernen der deutschen Sprache ist die Attraktivität des Studien- und Forschungsstandortes Deutschland, besonders im Bereich der international ausgerichteten ingenieurs- und naturwissenschaftlichen Studienprogramme, die zugleich eine kostengünstige Alternative zu den traditionell von Malaysiern bevorzugten Studienländern - USA, Großbritannien, Australien, Neuseeland - darstellen. Deutsch wird in Malaysia weder aus wissenschaftlichen Gründen oder als Kultursprache (wie etwa in Japan) noch aus wirtschaftlichen Erwägungen studiert, obgleich mehr als 400 deutsche Firmen im Land ansässig sind und deutsche Produkte ein hervorragendes Image haben (KÄRCHNER-OBER 2009).

Die Studie des Auswärtigen Amtes „Deutsch als Fremdsprache weltweit. Datenerhebung 2015“ kam zu dem Ergebnis, dass ,in Malaysia und Singapur [...] die Zahlen der Deutschlernenden bei niedrigen absoluten Zahlen um jeweils 77\% und 38\% gestiegen“ sind. Für Malaysia wurden für das Jahr 2014 etwa 8.200 Lernende (im Jahr 2010: 5.400) ausgewiesen - 3.900 an Universitäten/Colleges, 3.600 an Schulen und 750 am Goethe-Institut. Nach Einschätzung des Leiters der Sprachabteilung des Goethe-Instituts Malaysia, (E-mail vom 29.05.2017) lernten 2016 etwa 12.000 Personen in Malaysia die deutsche Sprache, u.a. am Goethe-Institut in Kuala Lumpur (950 Lernende, inklusive 29\% nicht-Malaysier z.B. aus 
Schaar, T.; Chang, S.W.; Gilgen, A. - "Magische Momente" im Klassenraum?

Ägypten, dem Iran, dem Irak, dem Jemen, dem Sudan, aus Vietnam, den Philippinen, aus Thailand...), am Institut der Deutsch-Malaysischen Gesellschaft in Penang, an 56 malaysischen Schulen, 6 internationalen Schulen (z.B. Deutsche Schule Kuala Lumpur, Französische Schule, Alice Smith School) sowie an 17 staatlichen und mehreren privaten Hochschulen und Colleges des Landes.

Die A-Level-(German)-Programme am International Education College (INTEC) der Universiti Teknologi MARA, Shah Alam und am German-Malaysian-Institute (GMI) Bangi, sowie das South-Australian-Matriculation-Programm am First City University College bereiten ihre Studierenden auf die Deutsche Sprachprüfung für den Hochschulzugang (DSH) bzw. auf TestDaF-Prüfungen (Niveau: B2/Selbständige Sprachverwendung und C1/Fachkundige Sprachkenntnisse) vor, die es den erfolgreichen Absolvent(inn)en schließlich ermöglichen, in Deutschland an ausgewählten Fachhochschulen ingenieurwissenschaftliche Programme auf Deutsch zu studieren. Universitäten und Schulen (mit Fremdsprachenunterricht) bieten Deutsch als elektive Kurse, als freiwilliges, studienbegleitendes Zusatzangebot bzw. berufsbegleitend/qualifizierend auf dem Niveau A1/Anfänger und A2/Grundlegende Kenntnisse an.

Neben dem Wahlfach Deutsch können bzw. konnten an der Universiti Malaya (UM) und an der Universiti Putra Malaysia (UPM) seit 1998 bzw. 2001 Bachelor-Programme mit Deutsch als Hauptfach (2015: 160 Studierende insgesamt) belegt werden: an der UM: der „Bachelor of Languages and Linguistics (German Language)“ und speziell zur Ausbildung von Deutschlehrern der „Bachelor of Languages and Linguistics (German with Education)“ - 2017 eingestellt - ; an der UPM: der „Bachelor of Arts in Foreign Languages (German)“.

Das Goethe-Institut Malaysia (gegründet 1962) unterrichtet in seinen Einrichtungen in Kuala Lumpur und Petaling Jaya intensive und extensive Deutschkurse auf allen Sprachlernniveaus. Zusätzlich gibt es spezielle Themenkurse für jugendliche Lerner, Kurse für Firmen oder Prüfungsvorbereitungskurse sowie Lehrerfortbildungen, Seminare und Workshops (http://www.goethe.de/ins/my/de/kua/lrn/deu.html).

Seit dem akademischen Jahr 2000/2001 besteht an der Fakultät für Moderne Sprachen und Kommunikation (FBMK) an der UPM das Bachelor of Arts in Foreign Languages (German)-Programm. Das ursprünglich als ein vierjähriger Studiengang konzipierte, aber aufgrund veränderter Richtlinien für Bachelor-Studiengänge auf sechs Semester verkürzte Programm sah als Studienziel für die „Nullanfänger“ das Erreichen des Sprachniveaus B1/Fortgeschrittene Sprachverwendung in Deutsch vor. Für die Mehrheit der malaysischen Studierenden ist Deutsch die dritte (L3) oder vierte Sprache (L4) nach Bahasa Malaysia, 
Schaar, T.; Chang, S.W.; Gilgen, A. - "Magische Momente" im Klassenraum?

Chinesisch, Tamil, Englisch (als Muttersprache) und Englisch (als Zweitsprache). Chinesische Malaysier sprechen oft noch verschiedene chinesische Dialekte wie Hokkien, Kantonesisch, Teochew und/oder Hakka (KÄRCHNER-OBER 2009).

2014 kam es im Bereich des BA German-Programms zu wesentlichen Neuerungen wie beispielsweise zur Einführung eines achtwöchigen Industriepraktikums (Industrial Training), eines Final Year Research Projects (BA Abschlussarbeit) sowie eines zusätzlichen siebten Semesters.

Von 2001 bis 2016 absolvierten 182 malaysische Studierende das BA GermanProgramm (122 credits) an der UPM - eine Kombination aus allgemeinem Sprachunterricht, traditionellen philologischen Inhalten und berufsvorbereitenden Kursen (68 credits): German Language I-III, Communication Skills I-II, Writing in German, German for Specific Purposes: Tourism / Commerce / Science and Technology, Historical Survey of Germany, Introduction to German Literature, Introduction to German Linguistics, Introduction to German Culture, 20th Century German Arts, Translation of Texts, Research Methodology in German, and Teaching German as a Foreign Language (Curriculum BA German, UPM). Mehrere Kurse wie Historical Survey of Germany, Introduction to German Literature, Introduction to German Linguistics und $20^{\text {th }}$ Century German Arts werden bilingual auf Deutsch und Englisch unterrichtet. UPM-Studierende belegen zusätzlich ein Nebenfach (30 credits), das entweder von der FBMK selbst (in Malay Literature, Communication, Arabic Language, English Literature, Chinese Literature, Translation and Interpretation) bzw. von anderen UPM-Fakultäten (z.B. Business Management, Resource Management, Business Administration, Hotel Management and Hospitality \& Recreation) angeboten wird. Jährlich beginnen etwa 30 Malaysier(innen) freiwillig oder zugewiesen - das Deutschstudium an der UPM.

Der Studiengang „Bachelor of Languages and Linguistics“ an der Fakultät für Sprachen und Linguistik an der UM hat eine stärkere germanistische Ausrichtung als das BA-GermanProgramm an der UPM. Zu den Kernfächern gehören allgemeine Sprachkenntnisse, Linguistik, Angewandte Linguistik sowie Literatur. Eine wissenschaftliche Abschlussarbeit im Semester 7 vervollständigt das Programm.

Ein vom Goethe-Institut, dem Institut Pendidikan Guru Kampus Bahasa Antarabangsa / International Languages Teacher Training Institute (IPGKBA) und dem malaysischen Hochschulministerium unterstütztes Twinning-Programm bereitete von 2007 bis 2017 insgesamt 75 malaysische Studierende in zwei Vorbereitungsjahren beim IPGKBA und in acht Semestern im Bachelor of Languages and Linguistics (German with Education)-Programm an 
Schaar, T.; Chang, S.W.; Gilgen, A. - "Magische Momente" im Klassenraum?

der UM auf den Beruf als Deutschlehrer an malaysischen Sekundarschulen (Day Schools) vor. Die Studienfächer konzentrierten sich auf den Spracherwerb auf der Fortgeschrittenenebene (B1-C1), auf (Angewandte) Linguistik und Literatur sowie auf Methoden und Didaktik des Unterrichts Deutsch als Fremdsprache (Curricula BA-German Programme, UM, zur Verfügung gestellt durch G. Schnieders, Email vom 16. Juni 2014).

Die im Auftrag des Ministry of Higher Education (MoHE) vorgelegte Studie „Future Direction of Language Education in Malaysia“ (2010) untersuchte den Status und die Inhalte der an malaysischen Universitäten angebotenen Linguistik-, Literatur- und Sprachprogramme, um Schlussfolgerungen für die künftige strukturelle, inhaltliche und marktorientierte Ausrichtung der Studienprogramme als auch für die Sprachenpolitik des Landes ziehen zu können. Durch die Analyse von unveröffentlichten Verbleibstudien der UM und UPM kam die MoHE-Studie zu der Schlussfolgerung, dass der malaysische Arbeitsmarkt den erfolgreichen Absolvent(inn)en von universitären sprach- und literaturwissenschaftlichen Programmen vielfältige Karrierechancen und -perspektiven in der Bildung, in der Industrie und im Finanzwesen sowie in der Öffentlichkeitsarbeit eröffne.

Eine UPM-geförderte Studie zu Berufsperspektiven der Absolvent(inn)en des BA German-Studienganges (2001-2014), an der sich mehr als 70\% der Graduierten beteiligten, kam in Übereinstimmung mit den Resultaten des MoHE zu dem positiven Ergebnis, dass die Mehrheit der Absolvent(inn)en (im Gegensatz zu vielen anderen Studiengängen an der Fakultät und Universität) keine Probleme hatte, bereits kurz nach dem Verlassen der Universität eine ihrer Qualifikation entsprechende Beschäftigung auf dem Arbeitsmarkt in Malaysia, vorrangig in administrativen Positionen (Sales, Marketing, Public Relations, Human Resources) in der Industrie und Finanzwirtschaft (54\%) zu finden. 26\% arbeiten als Lehrer(innen) an staatlichen oder privaten Schulen/Colleges und unterrichten Sprachen (Deutsch, Englisch, Mandarin, Malaysisch), aber auch naturwissenschaftliche Fächer. Etwa 10\% hatten sich für linguistische bzw. betriebswirtschaftliche Master-Programme (MBA) entschieden (SCHAAR et al. 2015, SCHAAR 2016; SCHAAR; OGASA 2017).

Im Jahr 2017 lehrten in Malaysia etwa 100 Sprachlehrer(innen) / Germanistik-, DaFDozent(inn)en die deutsche Sprache, aber auch Landeskunde, Linguistik, Literatur, Geschichte, Kunst bzw. Fachsprachen. Die 56 Deutschlehrer und -lehrerinnen an den Sekundarschulen repräsentieren die multi-ethnische Gesellschaft Malaysias, während die Mehrheit der Lehrkräfte am Goethe-Institut und an den Universitäten/Colleges aus Deutschland, der Schweiz und Österreich sowie aus anderen asiatischen, nordafrikanischen und europäischen Ländern wie 
Schaar, T.; Chang, S.W.; Gilgen, A. - "Magische Momente" im Klassenraum?

Ägypten, Bulgarien, Polen, Rumänien, Russland, der Slowakei, Tschechien, Thailand, der

Ukraine und Ungarn stammt. In nicht wenigen Fällen handelt es sich um „Quereinsteiger“ in die DaF-Lehrtätigkeit.

\section{Spielfilme im Unterricht Deutsch als Fremdsprache}

Deutschlehrende sollten stets darum bemüht sein, unter Einsatz vielfältiger Sozial- und Arbeitsformen, Lehrmittel und Medien einen studentenzentrierten, methodisch abwechslungsreichen, kommunikativen, handlungs-, standard- und kompetenzorientierten Unterricht zu gestalten, der den didaktisch-methodischen Prinzipien des Fremdsprachenunterrichts des Gemeinsamen Europäischen Referenzrahmen für Sprachen (GER) entspricht sowie eine auf Vertrauen, Fairness und Freude basierende motivierende Lernatmosphäre zu schaffen, in der autonomes, interaktives und interkulturell-orientiertes Lernen möglich, gefördert und gefordert wird. Studierende sollen Deutsch mit allen Sinnen erfahren und Lehrkräfte ihre curricular vorgegebenen Lehr- und Lernziele mit authentischen Inhalten wie Dokumentationen, Nachrichten, mit Landeskundlichem, mit Musik, touristischen Erkundungen, Ausstellungen, Kochstunden, Fotowettbewerben, künstlerischen Aktivitäten, mit Literatur, Theaterprojekten und Filmen anreichern.

Da Menschen ihre Lebenswirklichkeit bis zu 80\% visuell wahrnehmen (HARMS 2005: 247), Film als das Träger- und Vermittlungsmedium kulturspezifischer, soziologischer und historischer Inhalte identifiziert wurde und sich aus den Interdependenzen von Ton und Bild vielfältige interaktive Arbeitsmöglichkeiten und autonome Lernformen ergeben, sollte im modernen Fremdsprachenunterricht ein besonderes Augenmerk auf die Entwicklung des HörSeh-Verstehens sowie auf die Ausbildung der interkulturellen kommunikativen Kompetenz der Deutschstudierenden durch den Einsatz von vielfältigen filmischen Textsorten wie z.B. Spielfilmen, Dokumentar- und Kurzfilmen, Talkshows, Musikvideoclips, Sport- und Nachrichtensendungen, Werbespots gelegt werden (LAY 2009; ACHARYA 2010; FAISTAUER 2010; TONSERn 2010). Biechele (2010: 22) führt dazu aus:

Das Hör-Seh-Verstehen bezieht sich auf die Fertigkeit, audiovisuelle Medien, d.h. Filme unterschiedlicher Genres, im Fremdsprachenunterricht verstehen zu können. Gemäß dem semiotischen Status von Film bedeutet dies, Bild und Ton in ihren spezifischen Codes wahrzunehmen, zu verstehen und zu interpretieren. Das Hör-Seh-Verstehen zielt auf das adäquate Aufnehmen und Verarbeiten der kommunikativen Situation in ihrer Gesamtheit, d.h. das Erfassen der übermittelten Sprachzeichen und der nonverbalen wie extraverbalen, 
Schaar, T.; Chang, S.W.; Gilgen, A. - "Magische Momente" im Klassenraum?

kommunikative Funktion tragenden Informationen und deren intentionsgerechte, partnerbezogene und situationsgerechte Widerspiegelung und Interpretation.

2001 fand die audiovisuelle Rezeption auch Eingang in die Kompetenzbeschreibungen des Gemeinsamen Europäischen Referenzrahmens (GER) für Sprachen. In zahlreichen wissenschaftlichen Studien wurde der Einsatz von Spielfilmen als attraktives, modernes, motivierendes, emotional ansprechendes und effizientes (ergänzendes) Lehr- und Lernmedium im Sprachunterricht - das besonders dem Neugierverhalten junger Erwachsener entspricht, ihnen Spaß macht, die Imaginationsfähigkeit anspricht, Verstehensprozesse, Kompetenzen und „lebenswirkliches Lernen“ fördert sowie Reaktionen hervorruft - detailliert untersucht, die zahlreichen Vorteile herausgearbeitet und die Auswahlkriterien sowie die didaktische Vielfalt und ihre methodischen Prinzipien vor, während und nach dem Sehen beschrieben. Es wurde jedoch auch darauf verwiesen, dass eine unsachgemäße Implementierung des Mediums Film im Unterricht (d.h. das Anschauen eines Films oder von Filmsequenzen ohne ein erkennbares didaktisch-methodisches Konzept) - oft hervorgerufen durch mangelnde Kenntnisse der Lehrenden über die adäquate Verwendung von filmischen Textsorten und über vorbereitende, unterstützende und nachbereitende Aufgaben und Übungsformen - zu einer passiven Konsumentenhaltung der Lernenden, zu Frustrationen und/oder zum Verfehlen der eigentlichen Zielstellungen der Filmarbeit führen kann (BIECHELE 2006, 2007, 2010; BRANDI 1996; ChUdAK 2007, 2008, 2009, 2010; FAISTAUER 2010; GÜGOLD 1991; LAY 2009; RAABE 1997; SASS 2007; SCHWERDTFEGER 1989, 2003; STORK 2012; WELKE 2007, 2010).

King (2002) erläutert, dass die Wahl eines ansprechenden und geeigneten Films für eine spezifische Sprachlernergruppe, die den jeweiligen didaktisch-methodischen Zielstellungen Rechnung trägt, und sich auch für ein bestimmtes Sprachniveau eignet, für den Lehrenden und die Betrachter von größter Bedeutung ist. Der Schlüssel für eine erfolgversprechende Filmarbeit liegt in der Beachtung der folgenden Kriterien:

Filme im Fremdsprachenunterricht sollten aktuell und möglichst nicht älter als 15 Jahre alt sein. Sie müssen authentische, spannende, lebenswirklich relevante und für jugendliche Studierende interessante Geschichten erzählen, die ihre Filmsehgewohnheiten und Genrepräferenzen, ihr Allgemeinwissen, ihre Lebenserfahrungen, sowie ihre ethnisch-kulturellen und religiösen Hintergründe in Betracht ziehen. Die ausgewählten Spielfilme sollen die gesellschaftliche Realität in Deutschland /Österreich /der Schweiz, die Vielfalt von Kulturen und Lebensentwürfen, situativ bedingte Verhaltensweisen, Normen und Werte, sowie Alltagssituationen und Beziehungen von Menschen adäquat widerspiegeln. Filme, die lediglich kulturelle Stereotype vermitteln, müssen hingegen vermieden werden.

Filme spiegeln die Gesellschaft und die Zeit wider, in der sie entstanden sind. Sie bieten daher eine breite Basis zur Thematisierung sozialer Probleme einer Gesellschaft, z.B. 
Schaar, T.; Chang, S.W.; Gilgen, A. - "Magische Momente" im Klassenraum?

zwischenmenschliche Beziehungs- und Verhaltensmuster, Geschlechterrollen, Stellenwert von Familie und Peergroup, Identitätsmuster, Liebe, Glück und Unglück, Lebensziele, Ideale, Wertewandel, Traumklischees etc. Lay (2009: 109).

Spielfilme sollen Studierende in die Lage versetzen, komplexe gesellschaftliche, kulturelle und soziale Aspekte einer „fremden Kultur“ (in diesem Fall der deutschen/ österreichischen/ schweizerischen) differenzierend wahrzunehmen, diese kritisch zu analysieren und mit eigenen kulturellen Phänomenen in Beziehung zu setzen. Themen von aktuellem Interesse sind z.B.: Familie, Jugend, Alter, Konflikte zwischen den Generationen, Außenseiter, Minderheiten, Ausländer, Religionen, Migration, Wirtschaft, etc. So prägen Filme als authentische Kunstprodukte die Wahrnehmung und Deutung eigener kultureller Identität und bilden den Ausgangspunkt eines sensibleren Kulturverständnisses, das interkulturellen Missverständnissen und daraus resultierenden möglichen Konflikten vorbeugen kann (CHUDAK 2010; ETYA 2010; FAistauer 2010; GAMKRELIDZE 2010; TSCHUdinOWA 2010; TONSERN 2010).

Spielfilme sind Bestandteil jeder nationalen Kultur und dominante Ausdrucksform kultureller Identität, wobei die kulturelle Markiertheit sowohl den Inhalt eines Films als auch dessen Realisierung durch die filmischen Zeichen betrifft. Dadurch eröffnen sich Räume einer kulturspezifischen bzw. fremdkulturell bestimmten Wahrnehmung, die in konstruktiven, interaktiven und prozessorientierten Arbeitsformen produktiv gemacht werden können. Das Lernen mit Spielfilm/Kurzfilm im Unterricht DaF ist per se interkulturelles Lernen (BIECHELE 2010: 15).

King (2002), Lay (2009), Stork (2012) und Chudak (2010) legen dar, dass Lehrbüchern und Software zum Sprachenerwerb oft „Realität“ fehlt, diese stattdessen „künstliche“ Sprache in „künstlichen“ kommunikativen Situationen vorstellen und häufig Themen behandeln, die für die heutigen Studierenden von geringer Bedeutung sind. Filme im Sprachunterricht hingegen sind - trotz ihrer konstruierten fiktiven Realität - eine wissenschaftlich begründete und unterhaltsame Methode, um in „realistisch wirkende Kommunikation“ in „echterer“ Sprache und aktuelle Landeskunde einzuführen und die Lernenden zu befähigen, in authentischen Kommunikationssituationen adäquat handeln zu können. Stereotype, die DaF-Lehrwerke z.B. in Fotos und Illustrationen vermitteln, können durch Spielfilme relativiert werden. Indem sie „Sprache zum Leben erwecken“ und „Brücken in die reale Welt bauen“ kompensieren Spielfilme viele „Mängel“ in den lehrbuchorientierten und testgesteuerten Sprachklassenräumen wie z.B. monotone Grammatik-, Lese- und Hörübungen, uninspirierte 
Schaar, T.; Chang, S.W.; Gilgen, A. - "Magische Momente" im Klassenraum?

Rollenspiele etc. Die Studierenden sind Kommunikation in authentischer Sprache, den Stimmen verschiedener Muttersprachler, Dialekten und Akzenten ausgesetzt.

Spielfilme bieten schier endlose Möglichkeiten für den Sprach- und Kulturerwerb indem die Studierenden z.B. sehen, hören, sprechen, schreiben, deuten, erklären, interpretieren, assoziieren: d.h. sich kreativ mit der Zielsprache Deutsch auseinandersetzen, Aufgaben lösen, Materialien und Kritiken lesen, Wahrnehmungen und Konzepte vergleichen und diskutieren, Stimmungen und Gefühle reflektieren und ausdrücken, Dialoge, Plots, Einstellungen, Charaktere analysieren und z.B. in verschiedene Formen des Schreibens eingeführt werden: Zusammenfassungen, Biographien, Briefe, Charakterstudien (Beschreibungen), Vergleiche / Kontraste, Ursache / Wirkung Argumente, hypothetische Projektionen und Argumentationen (Diskussion über Vor- und Nachteile). Filme mit kontroversen Themen sind eine gute Grundlage für Argumentationen und Charakterstudien.

Die Studierenden machen sich zudem mit verschiedenen filmischen Textsorten, mit Spielfilmgenres / Subgenres - ihren einzigartigen Qualitäten und Charakteristiken - sowie mit Grundlagen der Filmanalyse - Kameraeinstellungen, -perspektiven und -bewegungen, Bildkomposition, Montage, Einstellungen, Schnitt, Toneffekte, Licht - vertraut. „Der gezielte Einsatz von Bildmedien im modernen Deutschunterricht fördert somit nicht nur die Verstehensund Sprachfertigkeit der Lernenden, sondern schärft auch ihren Blick für die Sprache der Bilder und baut auf diese Weise visuelle Literalität auf" (SASS 2007: 7). Lay (2009: 109) verweist zudem auf Informationsvermittlung durch „suprasegmentale (Intonation, Sprechpausen, Lautstärke, Zögern etc.) und paralinguistische (Gestik und Mimik) Faktoren, ... die Fremdsprachenlerner nutzen können, um Situationen adäquater einschätzen zu können.“ Die Studierenden erwerben so Medienkompetenz.

Die Verwendung von Spielfilmen bzw. Szenen / Sequenzen kann zyklisch progressiv erfolgen, d.h. bereits im Anfängerunterricht können Filme unter gewissen Aspekten (Vermittlung landeskundlicher Inhalte) z.B. mit englischen Untertiteln zum Einsatz kommen. Im Verlauf der sprachlichen Progression - B1, B2, C1 - werden zu den gleichen Filmen / Szenen sprachlich zunehmend anspruchsvollere Aufgaben bearbeitet (ACHARYA 2010; Biechele 2010; ETya 2010; Gamkrelidze 2010; SchaAR; LAPASAU; Ogasa 2013). Die Lernzielbereiche für Spielfilme im Fremdsprachenunterricht fasst Faistauer zusammen:

Hinsichtlich der Filmbildung im Fremdsprachenunterricht, die als ,Befähigung zu einem aktiverlebenden, kritisch und differenziert-wahrnehmenden, (inter)kulturell-sehenden und hörenden, selbstbestimmten und fachsprachlich-kreativen interkulturellen Handeln mit Filmen' gesehen 
Schaar, T.; Chang, S.W.; Gilgen, A. - "Magische Momente" im Klassenraum?

wird, sind Lernmöglichkeiten auf allen Ebenen und in allen Bereichen der Prinzipien möglich. Filmbildung erfolgt, wenn Lernende selbstbestimmt und aktiv Filme erleben, ihr Seh-Verstehen durch Rezeption schulen und durch Produktion verarbeiten, wenn ihr kritisches Denken durch unterschiedliche Gattungen und Genres von Filmen gefördert wird, wenn in anschließenden Aktivitäten an das Sehen filmische Inhalte sowohl auf mündlichem als auch auf schriftlichem Weg kritisch bewertet werden und dabei die eigene und die andere Kultur analysiert werden (2010: 44).

Eine erfolgreiche Filmarbeit bietet den Lernenden nicht nur ein emotionales Erlebnis, sondern vermittelt ihnen auch das Gefühl, kulturelle Erfahrungen gemacht, sich kritisch mit „anderen“ Denkweisen und Verhaltensmustern auseinandergesetzt und neue sprachliche Ausdrücke kennengelernt (und diese mündlich oder schriftlich angewendet) zu haben.

Als besonders geeignete Filme für den Fremdsprachenunterricht schlägt King (2002) romantische Komödien und wenig gewalttätige Actionfilme vor, in denen die Plots anregend, die Konflikte klar und einfach, die Protagonisten attraktiv und identifizierbar sind, und die Filmsprache zugänglich ist (Standardakzent).

\section{Zum Einsatz von Spielfilmen in außeruniversitären und universitären Deutschprogrammen in Malaysia}

In Ländern wie Malaysia, in denen der direkte Kontakt zur Zielsprache meist fehlt und im Wesentlichen auf das Klassenzimmer beschränkt bleibt, kommt Filmen als didaktischem Instrument im DaF/DaZ-Unterricht eine „Sonderrolle“ zu. Faistauer betont, dass für Lernende „,in anderen Kulturkreisen ... das Medium Film manchmal sogar der einzige Zugang zur visualisierten deutschsprachigen Welt" ist (2010: 34). Acharya charakterisiert Spielfilme als „,zusätzliche Vermittler von Kultur und Landeskunde“ und als „Treffpunkt zu einer ersten Begegnung und Auseinandersetzung mit der Vergangenheit und der Gegenwart der Zielkultur“‘ Durch Filme können die Lernenden z.B. „die geschichtlichen Ereignisse im Land der Zielsprache kennen lernen, miterleben bzw. nachempfinden“" (2010: 158).

Wie gestaltet sich nun die Situation hinsichtlich des Einsatzes von filmischen Textsorten in Malaysia? Sind Filme im L3-Deutschunterricht an malaysischen Sekundarschulen, Sprachinstituten, Colleges und Universitäten bereits zu einem „unbedingten Bestandteil eines zeitgemäßen Fremdsprachenunterrichts“ (SCHWERDTFEGER 2003: 299) geworden und werden die vielfältigen Lernangebote mit Film im Deutschunterricht nutzbar gemacht? Ist die Arbeit 
Schaar, T.; Chang, S.W.; Gilgen, A. - "Magische Momente" im Klassenraum?

mit Filmen bereits in der Aus- und Weiterbildung der Deutschlehrenden, z.B. durch das GoetheInstitut Malaysia verankert? Diesen Fragen möchte der vorliegende Text nachgehen.

43 in Malaysia tätige Deutschlehrende nahmen an einer im Februar/März 2016 durchgeführten empirischen Studie (Umfrage mittels Fragebögen, persönliche Interviews) - die sich methodologisch an Lays Artikel über die universitäre Filmarbeit in Taiwan (2009) orientierte - teil und lieferten Daten und Antworten, die sich auf vier wesentliche Bereiche konzentrierten - a) auf den Einsatz audiovisueller Medien im Deutschunterricht in Malaysia; b) auf die Rolle von Spielfilmen im Deutschunterricht allgemein; c) auf Motive zum Einsatz bzw. zum Nichteinsatz von Spielfilmen sowohl in Sprachkursen als auch in Fächern mit germanistischer, landeskundlicher, historischer oder künstlerischer Ausrichtung; und d) darauf, welche Filme unter welchen Zielstellungen im Unterricht gezeigt wurden. Die insgesamt hohe Teilnehmerquote, die etwa 50\% aller Deutschlehrenden in Malaysia repräsentierte, erlaubte eine zuverlässige Datenerhebung.

In einer elektronischen Umfrage (vierseitiger Fragebogen auf Deutsch / mit englischer Übersetzung) wurden die Lehrkräfte aufgefordert, 6 geschlossene Fragestellungen mit vorgegebenen möglichen Antworten durch Angabe von Prioritäten bzw. durch Anwendung der Fünf-Punkte-Likert-Skala positiv, neutral oder negativ zu bewerten. Die Antwortoptionen waren absteigend: 5 (Ich stimme voll zu), 4 (Ich stimme zu), 3 (unentschieden), 2 (Ich stimme nicht $\mathrm{zu}$ ), 1 (Ich stimme gar nicht zu). Während der persönlichen Befragung erhielten die Deutschlehrenden die Möglichkeit ihre Stellungnahme zu verschiedenen Aussagen zu begründen.

- Welche Medien/Medienträger werden überwiegend in Ihrem Deutschunterricht eingesetzt? (Prioritäten 1-3 eintragen)

- Was ist Ihre Perspektive hinsichtlich der Rolle von Medien und Spielfilmen im Deutschunterricht? (Mehrfachnennungen möglich)

- Welche audiovisuellen Medien setzen Sie im Unterricht ein? (Prioritäten 1-3 eintragen)

- Wie oft setzen Sie Spielfilme im DaF-Unterricht ein?

- Welche Zielstellungen verfolgen Sie mit dem Einsatz von Spielfilmen? (Mehrfachnennungen möglich)

- Warum setzen Sie im Unterricht keine oder nur selten Spielfilme ein? (Mehrfachnennungen möglich)

Hinzu kamen vier offene Fragen:

- Welche Spielfilme haben Sie in welchem Unterrichtsfach / Kurs gezeigt?

- Welche Spielfilme haben den Lernenden - Ihrer Meinung nach - am besten gefallen und warum? 
Schaar, T.; Chang, S.W.; Gilgen, A. - "Magische Momente" im Klassenraum?

- Haben Sie negative Reaktionen von Lernenden auf bestimmte Spielfilme erfahren? Wenn ja, auf welche und warum?

- Können Sie die Spielfilme unzensiert einsetzen? Falls nein, worauf müssen Sie besondere Rücksichten nehmen?

Ein positiv verlaufender Pretest mit den vier Deutschlehrenden an der UPM verdeutlichte nur geringfügige Designschwächen im ursprünglichen Fragebogen im Bereich der Auswahlantworten, die für die Umfrage entsprechend redigiert wurden. Die Darlegung der Ergebnisse folgt den Fragestellungen der Studie.

Anhand der demographischen Daten lässt sich feststellen, dass 42\% (18) der Befragten „umgeschulte“ malaysische Lehrerinnen und -lehrer waren, die Deutsch als Fremdsprache (A1, A2) an Sekundarschulen in den Provinzen/Territorien Kedah (4), Kuala Lumpur (2), Pahang (2), Perak (2), Penang (2), Perlis (1), Negeri Sembilan (2), Johor (2) and Selangor (1) lehrten. 10 Lehrkräfte (23\%) repräsentierten das German-Malaysian-Institute in Bangi (6) und das Goethe-Institut in Kuala Lumpur (4). 15 (35\%) arbeiteten als (Senior) Lecturers an fünf staatlichen Universitäten des Landes - UM (7), UPM (4), UMP (2), USM (1), UKM (1) - und unterrichteten u.a. in den beschriebenen Bachelor-Programmen Sprach- und Konversationskurse auf den Niveaustufen A1 bis C1 sowie germanistisch, didaktischmethodisch bzw. berufsvorbereitend ausgerichtete Fächer.

TABELLE 1

zeigt, welche Fächer / Kurse von den Befragten unterrichtet wurden

\begin{tabular}{ll} 
Unterrichtete Fächer & Lehrkräfte (n=43) \\
\hline Deutsche Sprache (A1-C1/GER) & $37(86.0 \%)$ \\
Linguistik & $5(11.6 \%)$ \\
Übersetzen & $4(9.3 \%)$ \\
Literatur & $3(6.9 \%)$ \\
Landeskunde & $3(6.9 \%)$ \\
Didaktik DaF & $3(6.9 \%)$ \\
Fachsprache Wirtschaft / Tourismus & $2(4.65 \%)$ \\
Deutsche Geschichte & $1(2.32 \%)$ \\
Deutsche Kunst & $1(2.32 \%)$ \\
\hline
\end{tabular}

Auf die Frage nach einem regelmäßigen Einsatz unterschiedlicher Medien / Medienträger im L3-Deutschuntericht in Malaysia gaben 39 Teilnehmer (91\%) an, (natürlich) die Deutsch-Lehrbücher (u.a. Studio D, Netzwerk), aber auch andere gedruckte Medien, sowie Computer (mit Projektoren) und das Internet zu integrieren. 34 (79\%) erwähnten auditive Medien wie CD-Spieler, 13 (30\%) arbeiteten mit Overhead-Projektoren, 5 (12\%) setzten 
Schaar, T.; Chang, S.W.; Gilgen, A. - "Magische Momente" im Klassenraum?

Fernsehgeräte mit DVD-Playern ein, und die 4 am Goethe-Institut Lehrenden (9\%) verfügten über und nutzten interaktive Whiteboards und deren Anwendungen.

Hinsichtlich der Perspektive der Deutsch-Lehrenden zur Rolle von Medien und Spielfilmen als Lehrmittel zeigt Tabelle 2, dass die Mehrheit die Wichtigkeit der Medienbildung im Unterricht (theoretisch) bestätigte, dass Filme als ein modernes, lebensbezogenes, emotionales, motivierendes und z.B. das interkulturelle Verständnis förderndes Lehr- und Lernmedium verstanden, und dass die Entwicklung des Hör-SehVerstehens sowie die Befähigung der Lernenden zur Versprachlichung der Inhalte als die eigentliche Kompetenz des Fremdsprachenunterrichts angesehen wurden. Weder Zustimmung noch Ablehnung, also eher Unsicherheit und fehlende Erfahrung im Umgang mit filmischen Textsorten zeigte sich sowohl bei Sekundarschul-, Goethe-Institut- und Collegelehrkräften (SGICL) als auch bei Universitätsdozent(inn)en (UD) bei der Einschätzung der Wertigkeit von Filmen und/oder Lehrwerken bei der Vermittlung von Landeskunde und Kulturwissen sowie hinsichtlich des Alters der im Unterricht zum Einsatz kommenden Filme (Tabelle 2). Über die Beurteilung des Einsatzes von Filmen im L3-Anfängerunterricht (Deutsch) und über das Zuschalten englischer Untertitel zur Unterstützung des Verständnisses des Gezeigten im L3Anfängerunterricht bestanden hingegen Meinungsverschiedenheiten. 64\% der SGICL - aber nur 20\% der UD - lehnten den Einsatz von fiktionalen Filmen im DaF-Unterricht entweder völlig ab oder waren eher unentschieden. Während Lehrkräfte an Universitäten englische Untertitel (wohl aus der Unterrichtserfahrung heraus) vorbehaltlos bejahten, waren SGICL eher unentschieden.

\section{TABELLE 2}

Zur Rolle von Medien und Spielfilmen im Deutschunterricht

Perspektive der Lehrkräfte zur Rolle von Medien und Spielfilmen im Deutschunterricht
Goethe-Institut Universitäten GMI, Schulen $\mathrm{n}=28 \quad \mathrm{n}=15$

\begin{tabular}{lcc}
\hline Medienerziehung ist im Fremdsprachenunterricht wichtig. & $\begin{array}{c}4.24 \\
\text { Ich stimme zu }\end{array}$ & $\begin{array}{c}4.52 \\
\text { Ich stimme voll zu }\end{array}$ \\
\hline $\begin{array}{l}\text { Spielfilm ist ein attraktives, lebensbezogenes, emotionales, } \\
\text { motivierendes Lehr- und Lernmittel. }\end{array}$ & $\begin{array}{c}4.2 \\
\text { Ich stimme zu }\end{array}$ & $\begin{array}{c}4.36 \\
\text { Ich stimme zu }\end{array}$ \\
\hline $\begin{array}{l}\text { Das Lernen mit Spielfilmen ist per se interkulturelles Lernen. } \\
\text { Ich stimme zu }\end{array}$ & $\begin{array}{c}4.16 \\
\text { Ich stimme zu }\end{array}$ \\
\hline $\begin{array}{l}\text { Das Hör-Sehverstehen ist die eigentliche grundlegende } \\
\text { Kompetenz des Fremdsprachenlernens. }\end{array}$ & $\begin{array}{c}4.08 \\
\text { Ich stimme zu }\end{array}$ & $\begin{array}{c}3.71 \\
\text { Ich stimme zu }\end{array}$ \\
\hline $\begin{array}{l}\text { Arbeit mit Spielfilmen ist nicht für den Anfängerunterricht } \\
\text { geeignet. }\end{array}$ & $\begin{array}{c}2.96 \\
\text { unentschieden }\end{array}$ & $\begin{array}{c}2.35 \\
\text { ich stimme nicht zu }\end{array}$
\end{tabular}


Schaar, T.; Chang, S.W.; Gilgen, A. - "Magische Momente" im Klassenraum?

Spielfilme sind für Studierende der wichtigste Zugang zum

Kulturwissen unserer Zeit.

3.48

3.44

Spielfilme sind für die Vermittlung von Landeskunde wichtiger als Lehrbuchtexte.

unentschieden unentschieden

Spielfilme können im Deutschunterricht nur eine Ergänzung zu

Lehrbüchern sein.

3.4

3.07

unentschieden unentschieden

Spielfilme sollten nicht älter als 15 Jahre alt sein, um noch

didaktische Funktionen im Unterricht erfüllen zu können.

Deutsche Spielfilme im Anfängerunterricht können z.B.

englische Untertitel haben.

$3.2 \quad 3.4$

unentschieden unentschieden

$2.96 \quad 2.79$

unentschieden unentschieden

$3.48 \quad 4.57$

unentschieden Ich stimme voll zu

Tabelle 3 impliziert, dass mehr als die Hälfte aller Befragten Musikclips und Kurzfilme in ihre Lehrtätigkeiten integriert hatten. Immerhin zeigten auch 18 (41,8\%) der Lehrkräfte deutsche Spielfilme. UD schienen Spielfilme gegenüber anderen audiovisuellen Medien deutlich zu bevorzugen, während SGICL Kurzfilme und Musikclips favorisierten. Die eher positive Aussage über den Einsatz von Spielfilmen im Unterricht muss allerdings dahingehend relativiert werden, dass $14(77,7 \%)$ dieser 18 Lehrkräfte lediglich einen Spielfilm pro akademischem Jahr / Sprachkurs bzw. während ihrer gesamten Lehrtätigkeit gezeigt hatten. Nur 4 der 11 UD (36,6\%) lieferten 3-5 „magische Momente“ in ihren Kursen, während zwei UPM-Dozent(inn)en (18\%) mehr als fünf Spielfilme zeigten. Mehr als die Hälfte der Befragten hingegen $(25=58 \%)$ - vor allem SGICL (z. B. alle 6 Teilnehmer vom GMI!) - hatten die umfangreichen didaktisch-methodischen Anwendungsmöglichkeiten von Spielfilmen im modernen Sprachunterricht bislang völlig ignoriert.

TABELLE 3

Audiovisuelle Medien im Deutschunterricht in Malaysia

\begin{tabular}{llll} 
& Goethe-Institut/GMI/Schulen & Universitäten & gesamt \\
\hline Teilnehmer & $\mathbf{2 8}$ & $\mathbf{1 5}$ & $\mathbf{4 3}$ \\
Spielfilme & $7(25,0 \%)$ & $11(73,3 \%)$ & $\mathbf{1 8}(41,8 \%)$ \\
Dokumentationen & $12(42,8 \%)$ & $2(13,3 \%)$ & $14(32,5 \%)$ \\
Kurzfilme & $18(64,2 \%)$ & $4(26,6 \%)$ & $22(51,1 \%)$ \\
Musikclips & $18(64,2 \%)$ & $5(33,3 \%)$ & $23(53,4 \%)$ \\
Werbespots & $12(42,8 \%)$ & $5(33,3 \%)$ & $17(39,5 \%)$ \\
\hline
\end{tabular}

Dass Sekundarschullehrkräfte weitgehend auf Spielfilme im Unterricht verzichteten, hat verschiedene Gründe. Kärchner-Ober verwies in ihren Untersuchungen auf südostasiatische L2/L3-Lerntraditionen, in denen das Memorieren, Wörter übersetzen sowie eine starke Fokussierung auf Grammatik dominieren und fremdkulturelle Komponenten im Unterrichtsmaterial weitgehend fehlen (KÄRCHNER-OBER 2009). 
Schaar, T.; Chang, S.W.; Gilgen, A. - "Magische Momente" im Klassenraum?

Die gegenwärtige Bildungspolitik Malaysias konzentriert sich eher auf die Entwicklung von Informationskompetenz, d.h. auf das Suchen, Finden und Bewerten von Informationen insbesondere im Internet, als auf eine umfassende, den Erfordernissen des 21. Jahrhunderts entsprechende Medienbildung. Obgleich Medien von signifikanter Bedeutung für die junge Generation Malaysias sind, spielt die Entwicklung einer kritischen Medienkompetenz, das Wissen um die Komplexität von Medien, Zeichen, Texten, Institutionen, Rezipienten und Reaktionen in schulischen Lehrplänen keine Rolle (SHANTHI; KHOO 2006; PRASAD; RAO; DOLlaH 2012; PrASAD; SHANTI 2013):

Learning in Malaysia however continues to render visual media study invisible within the formal primary and secondary school settings and there is a clear case for media literacy to be developed in ways that enable young people to comprehend daily life and media experiences critically as well as to engage actively and creatively in the various spheres. As such, it is apparent that not much effort has been put by either government, media institutions or schools in Malaysia in addressing this issue faced by the student community. Another issue would be how to develop media literacy skills among the students in Malaysia, where there is hardly any room for introducing media education in the school curriculum (SHANTHI; KHOO 2006: 1).

Außerdem muss eingeschätzt werden, dass das Sprachniveau der ,alten“ DaFSekundarschullehrer, die seit Mitte der 1990er Jahre vom Goethe-Institut von anderen Unterrichtsfächern auf Deutsch als Fremdsprache „umgeschult“ worden waren und gegenwärtig A1 und A2 unterrichten, immer noch kaum mehr als B1 beträgt, was für die kritische Rezeption und den gezielten didaktisch-methodischen Einsatz von deutschen Spielfilmen im Unterricht faktisch nicht ausreicht. Hinzu kommt, dass Fortbildungsangebote zur Implementierung moderner Medien im Fremdsprachenunterricht in Malaysia kaum existieren. So hat das Goethe-Institut Malaysia in den vergangenen Jahren lediglich einen Workshop für Sekundarschullehrer zum Einsatz von Kurz- und Spielfilmen im L3Deutschunterricht veranstaltet (April 2014). Für an Hochschulen Lehrende gab es bisher keine Veranstaltungen.

Wenn Spielfilme im L3-Deutschunterricht in Malaysia überhaupt zum Einsatz kamen (Tabelle 4), dann dienten diese vor allem der Unterhaltung und der (vorgeblichen) Motivierung der Lernenden. Als Lernzielbereiche nannten die Befragten aber auch die Vermittlung landeskundlicher fremdkultureller Aspekte (durch Aufnahme und Verarbeitung von Informationen aus Filmsequenzen), die Entwicklung des Hör-Seh-Verstehen inklusive der Sprachaufmerksamkeit und das Training des verstehenden Hörens. In nur wenigen Fällen wurde(n) literarische Werke und Ereignisse der deutschen Geschichte visualisiert und filmisch- 
Schaar, T.; Chang, S.W.; Gilgen, A. - "Magische Momente" im Klassenraum?

ästhetische Kompetenz bzw. Filmkunst/-wissen (Regisseure, Genres, Epochen, filmische Entwicklungen) vermittelt.

TABELLE 4

Warum werden Spielfilme im Deutschunterricht eingesetzt?

\begin{tabular}{|c|c|c|}
\hline $\begin{array}{l}\text { Warum setzen Sie Spielfilme ein? (Mehrfachnennungen } \\
\text { möglich) } \mathbf{n}=\mathbf{1 8} \\
\text { Zur: }\end{array}$ & $\begin{array}{l}\text { Goethe-Institut, } \\
\text { GMI, Schulen } \\
\mathrm{n}=7\end{array}$ & $\begin{array}{l}\text { Universitäten } \\
\mathrm{n}=11\end{array}$ \\
\hline Unterhaltung, Anregung und Motivation & $6(85,7 \%)$ & $8(73 \%)$ \\
\hline $\begin{array}{l}\text { Vermittlung von (mehr) authentischen sprachlichen } \\
\text { Aspekten }\end{array}$ & $5(71,4 \%)$ & $7(64 \%)$ \\
\hline Entwicklung des Hör-Seh-Verstehens & $3(42,8 \%)$ & $5(45,5 \%)$ \\
\hline Vermittlung von Aspekten der deutschen (Alltags-)Kultur & $4(57,1 \%)$ & $6(54,5 \%)$ \\
\hline Vermittlung von Aspekten der deutschen (Jugend-)Kultur & $2(28,5 \%)$ & $2(18 \%)$ \\
\hline $\begin{array}{l}\text { Vermittlung von Aspekten der multikulturellen Gesellschaft } \\
\text { Deutschlands }\end{array}$ & $3(42,8 \%)$ & $4(36 \%)$ \\
\hline $\begin{array}{l}\text { Möglichkeit des Kulturvergleichs und des Bewusstmachens } \\
\text { der „eigenen Kultur“ }\end{array}$ & $5(71,4 \%)$ & $3(27 \%)$ \\
\hline Visualisierung von Werken der deutschen Literatur & $1(14,2 \%)$ & - \\
\hline Visualisierung des Lebens von berühmten Deutschen & - & - \\
\hline Vermittlung von deutscher Geschichte & $2(28,5 \%)$ & $1(9 \%)$ \\
\hline Vermittlung von Filmwissen und Medienkompetenz & $2(28,5 \%)$ & $1(9 \%)$ \\
\hline Übersetzung von Texten & - & $1(9 \%)$ \\
\hline aus anderen Gründen: (genannt: Filmrezension schreiben) & & $1(9 \%)$ \\
\hline
\end{tabular}

Auf die Frage nach den Gründen, warum Spielfilme in ihrem DaF-Unterricht keine oder fast keine Rolle spielten (siehe Tabelle 5), nannten 25 der befragten Deutschlehrenden vor allem fehlendes filmdidaktisches und filmästhetisches Wissen (Medienkompetenz), den hohen zeitlichen Aufwand bei der Auswahl und bei der didaktisch-methodischen Auf- und Nachbereitung von Spielfilmen sowie teilweise fehlende technische Voraussetzungen (besonders an Sekundarschulen). Die Hälfte der UD gaben an, dass Filme nicht zu ihren Unterrichtsinhalten passen würden und ein Drittel der SGICL waren der Meinung, dass die authentische Filmsprache die Lernenden überfordern und somit demotivierend wirken würde. $17(68 \%)$ der Befragten erklärten zudem, dass der Unterrichtsfokus nicht primär auf der Vermittlung „lebendiger Sprache“, sondern auf der Befähigung der Lernenden zum Bestehen verschiedener Sprachprüfungen mittels traditioneller Lehr-, Lern- und Übungsformen liegen würde und der sich daraus ergebende „Zeitdruck“ den Einsatz von Spielfilmen kaum zulasse. 
Schaar, T.; Chang, S.W.; Gilgen, A. - "Magische Momente" im Klassenraum?

TABELLE 5

Warum werden Spielfilme nicht oder selten eingesetzt?

\begin{tabular}{|c|c|c|}
\hline $\begin{array}{l}\text { Warum setzen Sie im Unterricht keine (oder nur selten) Filme } \\
\text { ein? } \\
\text { (Mehrfachnennungen möglich) } \mathbf{n}=\mathbf{2 5}\end{array}$ & $\begin{array}{l}\text { Schulen, GMI } \\
\text { Goethe-Institut } \\
\quad \mathrm{n}=15\end{array}$ & $\begin{array}{l}\text { Universitäten } \\
\qquad \mathrm{n}=10\end{array}$ \\
\hline $\begin{array}{l}\text { Die Auswahl des geeigneten Films in Relation zu den } \\
\text { Unterrichtszielen ist zu aufwändig. }\end{array}$ & $2(13,3 \%)$ & $3(30 \%)$ \\
\hline $\begin{array}{l}\text { Die didaktische Vorbereitung in Relation zu den Unterrichtszielen } \\
\text { ist zu aufwändig. }\end{array}$ & $2(13,3 \%)$ & $8(80 \%)$ \\
\hline $\begin{array}{l}\text { Das Vorbereiten, Zeigen und Nachbearbeiten des Films nimmt zu } \\
\text { viel Zeit in Anspruch. }\end{array}$ & $6(40 \%)$ & $3(30 \%)$ \\
\hline $\begin{array}{l}\text { Ich verfüge selbst nicht über genügend filmdidaktisches Wissen, } \\
\text { um einen qualitativ hochwertigen Unterricht zu gestalten. }\end{array}$ & $7(46,6 \%)$ & $6(60 \%)$ \\
\hline $\begin{array}{l}\text { Ich verstehe nicht genug von Dramaturgie, Kameraführung, Ton, } \\
\text { Licht und Montage. }\end{array}$ & $5(33,3 \%)$ & - \\
\hline $\begin{array}{l}\text { Meine Studierenden verfügen nicht über genügend Medienbildung, } \\
\text { Weltwissen und Kulturverständnis, um einen Film den } \\
\text { Unterrichtszielen entsprechend analysieren zu können. }\end{array}$ & $3(20 \%)$ & - \\
\hline $\begin{array}{l}\text { Die technischen Möglichkeiten sind nicht gegeben (Ausstattung der } \\
\text { Klassenräume). }\end{array}$ & $5(33,3 \%)$ & - \\
\hline Filme passen nicht zu meinen Unterrichtsinhalten. & $3(20 \%)$ & $5(50 \%)$ \\
\hline Spielfilme sind wegen ihrer Länge ungeeignet. & $4(26,6 \%)$ & $1(10 \%)$ \\
\hline Ich überlasse die Filmarbeit meinen Kolleg(inn)en. & $2(13,3 \%)$ & $1(10 \%)$ \\
\hline $\begin{array}{l}\text { Ich betrachte Filmarbeit lediglich als „Lückenfüller“ oder } \\
\text { „Belohnung“. }\end{array}$ & $3(20 \%)$ & - \\
\hline $\begin{array}{l}\text { Die Sprache des Films ist zu kompliziert für die Studierenden } \\
\text { (selbst mit Untertiteln). }\end{array}$ & $5(33,3 \%)$ & - \\
\hline Ich schaue selbst nicht gern Filme. & $2(13,3 \%)$ & - \\
\hline $\begin{array}{l}\text { Studierende schauen schon genug Filme in der Freizeit, daher ist es } \\
\text { nicht notwendig, auch im Unterricht Filme einzusetzen. }\end{array}$ & - & - \\
\hline
\end{tabular}

Die Mehrzahl der deutschen Spielfilme - siehe Tabelle 6 - wurde während der regulären Unterrichtsstunden bzw. im Rahmen curricular verankerter interkultureller Workshops (INTEC, GMI) gezeigt. Die Lehrkräfte verwendeten in der Regel DVDs, die für Lehrveranstaltungen oder die gemeinnützige Öffentlichkeitsarbeit von der Filmabteilung des Goethe-Instituts in München zugelassen sind und den Abteilungen / Schulen vom GoetheInstitut in Kuala Lumpur oder über den Deutschen Akademischen Austauschdienst (DAAD) zur Verfügung gestellt wurden. Einige benutzten auch Spielfilme aus privaten Sammlungen. Bei der Auswahl und beim Vorführen von Spielfilmen im Unterricht in Malaysia - einem überwiegend islamisch geprägten Land - mussten die Lehrkräfte besondere Rücksicht auf religiöse Inhalte nehmen sowie Nackt/Liebesszenen (möglichst) „zensieren“, d.h. eine entsprechende „Warnung“ an die Zuschauer geben, den Projektor abdecken oder die Szene(n) 
Schaar, T.; Chang, S.W.; Gilgen, A. - „Magische Momente" im Klassenraum?

auslassen. Filme, in denen Homosexualität oder Transgender thematisiert werden, sind in Malaysia verboten.

TABELLE 6

Filme, die seit 2010 im DaF-Unterricht/in Workshops gezeigt wurden

\begin{tabular}{l|l} 
Sprachkurse / Fächer (Niveau GER) & Filmtitel (ohne Angaben zu Regisseuren / Erscheinungsjahr)
\end{tabular}

Goethe-Institut, GMI, Schulen:

Allgemeine Sprachkurse (A1/A2)

Alle Filme wurden mit englischen Untertiteln gezeigt, da das Sprachniveau für das

Erfassen der Inhalte nicht ausreichte.

\section{Universitäten:}

Allgemeine Sprachkurse (A1-C1)
Barfu $\beta$, Das Experiment, Das fliegende Klassenzimmer, Der ganz große Traum, Good bye, Lenin, Hanni und Nanni, Hexe Lili, Jenseits der Stille, Kebab Connection, Das Leben der Anderen, Lola rennt, Napola, Neukölln Unlimited, Die Rote Zora, Der Teufelskicker, Das Wunder von Bern

12 Winter, Barfu $\beta$, Bella Martha, Blueprint, Dschungelkind, Fack Ju, Göthe, Die Fremde, Der ganz große Traum, Good bye, Lenin, Im Juli, In drei Tagen bist du tot, Jenseits der Stille, Keinohrhasen, Kokowääh, Kokowääh 2, Lola rennt, Lila Lila, Kebab Connection, Knocking on Heaven's Door, Renn, wenn du kannst, Rubbeldiekatz, Same Same, but Different, Schlussmacher, Türkisch für Anfänger, Was tun, wenn's brennt, What a Man, Who am I? Das Wunder von Bern

\begin{tabular}{|c|c|}
\hline $\begin{array}{l}\text { Landeskunde (B1) } \\
\text { mit deutschen Untertiteln }\end{array}$ & $\begin{array}{l}\text { Kirschblïten - Hanami, Almanya-Willkommen in } \\
\text { Deutschland, Solino, Eltern }\end{array}$ \\
\hline $\begin{array}{l}\text { Deutsche Geschichte (A2) } \\
\text { Der Kurs wird auf Englisch unterrichtet. Alle } \\
\text { Filme wurden mit englischen Untertiteln } \\
\text { gezeigt, da das Sprachniveau A2 für das } \\
\text { Erfassen der Inhalte nicht ausreicht. }\end{array}$ & $\begin{array}{l}\text { deutsche Spielfilme: Der ganz große Traum, Stalingrad, } \\
\text { Napola, Unsere Mütter. Unsere Väter, Die Weiße Rose, } \\
\text { Sophie Scholl. Die letzten Tage, Dresden, Hitlerjunge } \\
\text { Salomon, Der Untergang, Lore, Anonyma - Eine Frau in } \\
\text { Berlin, Das Wunder von Bern, Der Tunnel, Sonnenallee, } \\
\text { Nikolaikirche, Das Wunder von Berlin, Der Baader-Meinhof- } \\
\text { Komplex, Das Leben der Anderen } \\
\text { nichtdeutsche Spielfilme: Luther, All Quiet on the Western } \\
\text { Front, Stalingrad, Süskind, The Grey Zone, The Pianist, } \\
\text { Colette - Prisoners of Auschwitz }\end{array}$ \\
\hline $\begin{array}{l}\text { Deutsche Literatur (A2, B1) } \\
\text { Alle Filme wurden mit englischen Untertiteln } \\
\text { gezeigt, da das Sprachniveau für das } \\
\text { Erfassen der Inhalte nicht ausreichte. }\end{array}$ & $\begin{array}{l}\text { Die Nibelungen. Der Fluch des Drachen, Woyzeck, Der } \\
\text { Schimmelreiter, Goethe! Schiller }\end{array}$ \\
\hline $\begin{array}{l}\text { Deutsche Kunst (B1/B2) } \\
\text { Die fünf erstgenannten sind „, Stummfilme“ } \\
\text { mit kurzen deutschen Zwischentexten. }\end{array}$ & $\begin{array}{l}\text { Das Cabinet des Dr. Caligari, Faust, Nosferatu, Die } \\
\text { Nibelungen, Metropolis, Comedian Harmonists }\end{array}$ \\
\hline
\end{tabular}

Die offenen Fragen nach positiven / negativen Reaktionen der Studierenden auf bestimmte Spielfilme und Gründen dafür wurden leider sehr unzureichend, und häufig lediglich in Stichpunkten beantwortet. Es lassen sich somit nur folgende Tendenzen erkennen:

Die Komödien (Kokowääh, Barfuß, Knockin on Heaven's Door, Kebab Connection, Schlussmacher, Fack Ju, Göthe u.a. - siehe Tabelle 9) hätten nach Aussagen der Lehrenden 
Schaar, T.; Chang, S.W.; Gilgen, A. - "Magische Momente" im Klassenraum?

besonderen Anklang gefunden, da „die Geschichten in schönen Bildern produziert, leicht nachvollziehbar, sinnvoll und lustig“ waren und sich die Zuschauer mit den jugendlichen Protagonisten identifizieren konnten. Who am I? (2014, Baran bo Odar) entsprach am ehesten den Hollywood-geprägten Filmsehgewohnheiten der Studierenden und überzeugte durch Action und Spannung, interessante Charaktere, schnelle Schnitte und einen ansprechenden Soundtrack. Die Themen Liebe, Partnerschaft, Familie, Konflikte zwischen Generationen, Musik (Jenseits der Stille), studentisches Leben (Renn, wenn du kannst), Ankunft und Erfahrungen in der Fremde (Almanya - Willkommen in Deutschland, Solino) wären für viele Lernende lebenswirklich relevant gewesen.

Motivierend hätte gewirkt, wenn die im Film gesprochene Sprache (Wörter, Wortgruppen, Sätze) als „,bereits gelernt“ identifiziert und verstanden wurde. Das Coming-ofAge / Familien-Drama Jenseits der Stille (1996, Caroline Link) wurde im Top-20 Ranking (Tabelle 9) als Nr. 6 relativ hoch eingestuft, weil die Darstellung des Familienlebens und der Probleme zwischen den behinderten Eltern und den nichtbehinderten Töchtern als „sehr realistisch" erschien.

Problematisch gestaltete sich der Einsatz von historischen Spielfilmen (Das Leben der Anderen, Good bye, Lenin) zur Visualisierung von wesentlichen Ereignissen der Geschichte der Deutschen Demokratischen Republik im allgemeinen Sprachunterricht insbesondere an Sekundarschulen. Wie in Interviews festgestellt wurde, hat sich die Vorführung des Oscar gekrönten Films Das Leben der Anderen (2006, Florian Henckel von Donnersmarck) - über die Überwachung eines ostdeutschen Autors und seiner Freunde durch die Staatssicherheit ohne eine lernergerechte Einführung in die umfassenden historischen Hintergründe (vor dem Sehen), ohne gezielte Leitfragen (während des Sehens) und ohne eingehende und vertiefende Diskussionsrunden (nach dem Sehen) zu einer frustrierenden Erfahrung für die Schüler gestaltet. Die Lehrerin beklagte: „Obwohl ich den Schülern mündlich einige Einblicke in die DDR und in das SED-Regime gegeben habe, haben sie den Film überhaupt nicht verstanden!“ Wenn ein anderer Sekundarschullehrer darlegte, dass der Film Good bye, Lenin (2003, Wolfgang Becker) von seinen 14jährigen Schülern „sehr positiv“ wahrgenommen wurde, da „die komplexe politische Situation in Ostdeutschland in den achtziger Jahren, der Fall der Berliner Mauer und die Probleme der Ostdeutschen“, die sich nach der deutschen Wiedervereinigung mit einem völlig veränderten politischen und wirtschaftlichen System auseinandersetzen mussten, „humorvoll und spannend dargestellt“ wurden, ist man versucht, davon auszugehen, dass diese Aussage eher seine eigene Wahrnehmung widerspiegelt, als die 
Schaar, T.; Chang, S.W.; Gilgen, A. - "Magische Momente" im Klassenraum?

der malaysischen Jugendlichen, die entweder nur begrenzte oder gar keine Kenntnisse von dieser dramatischen und wichtigen Periode in der deutschen Geschichte haben. Anhand dieser zwei Beispiele lässt sich vermuten, dass historische Spielfilme im Anfängerunterricht DaF eher zur Unterhaltung der Lernenden (und Lehrenden) als zur Vermittlung historischer Ereignisse oder gar zur Entwicklung von historischer Medienkompetenz eingesetzt wurden.

Die Filme Das Wunder von Bern (2003, Sönke Wortmann) und Der ganz große Traum (2011, Sebastian Grobler) hingegen bieten neben historischem Ambiente vor allem Einblicke in die Geschichte und in die Rolle des Fußballs in Deutschland: die an Konflikten und Widerständen reiche Einführung des Spiels durch den Gymnasiallehrer Konrad Koch im Jahre 1874 in Braunschweig; und Westdeutschlands unerwarteter Sieg bei der Weltmeisterschaft 1954 in Bern vor dem Hintergrund der Wiedergeburt des Landes nach der Niederlage im Zweiten Weltkrieg. Diese Edutainment-Produkte haben malaysischen Lernenden und Lehrenden, die oft fußballbegeistert sind und über gefestigtes Fußballwissen verfügen, gleichermaßen gefallen.

SGICL/UD berichteten, dass ihre Lernenden auf deutsche Spielfilme „negativ“ reagierten - d.h. das Interesse an ihnen verloren - wenn sie länger als 90 Minuten dauerten, wenn „zu schnell gesprochen wurde“ und wenn die in den Filmen dargestellten Themen „uninteressant“ und ,irrelevant“ und somit „langweilig“ waren. Die Oertel/Deppe-Verfilmung der Novelle „Der Schimmelreiter“ von Theodor Storm aus dem Jahre 1933 - eingesetzt im Literaturkurs an der UPM (schwarz-weiß, VHS Kassette) - wurde für die Lernenden zu einem wahren Albtraum. Selbst die Dozentin empfand den Film als „fad“ und „trübsinnig“ (setzte diesen dennoch ein).

Unzensiert gezeigte Nackt-/Liebesszenen hätten - aufgrund der konservativen Prägung malaysischer Jugendlicher - peinlich berührtes Gekicher oder offene Empörung (Verlassen des Klassenraumes) hervorgerufen.

Nach Einschätzung der Lehrkräfte, die Spielfilme bzw. Filmszenen z.B. zur Entwicklung der Fertigkeit Schreiben einsetzten, bewährten sich Didaktisierungsmittel wie Charakterisierungen von Figuren / Figurenkonstellationen und ihren Beziehungen zueinander sowie Beschreibungen von Situationen, von Orten, Räumen und Landschaften. Filmische Redemittel wurden syntaktisch und semantisch analysiert und in grammatischen Übungen angewendet und gefestigt. Basierend auf den in Filmen wahrgenommenen Sprachstrukturen produzierten die Lernenden selbst verschiedene Textsorten wie Dialoge, Meinungsäußerungen, Stellungnahmen, fiktive Lebensläufe. 
Schaar, T.; Chang, S.W.; Gilgen, A. - "Magische Momente" im Klassenraum?

Im akademischen Jahr 2015/2016 sahen die BA-German Studierenden des 5. Semesters im Kurs BBD 4409 Communication Skills II (Level B1/B2) die Spielfilme Kokowääh, Barfuß, Jenseits der Stille, Die Fremde, Kebab Connection, Same Same, But Different, Eltern, Was bleibt?, Türkisch für Anfänger, und Renn, wenn du kannst mit deutschen Untertiteln. Die Filme wurden filmspezifisch analysiert (Kameraeinstellungen und Kameraperspektiven) und verschiedene kulturelle Aspekte (Freundschaft, Integration von Ausländern, Jugend, Familie / Patchwork-Familie, Alter, Menschen mit Behinderungen, Ehe mit Ausländern, Umgang mit Krankheit / Verlust / Tod etc.) miteinander verglichen und mündlich / schriftlich diskutiert. Darüber hinaus spielten die Studentinnen und Studenten Filmszenen nach, schrieben alternative Dialoge, Szenen und Enden, erstellten Filmkritiken, führten fiktive Interviews mit den Charakteren, schlüpften in die Rolle von Charakteren und erläuterten ihre Gedanken (point-ofview). Sie drehten selbst kleine Filme (mit Mobiltelefonen) und beschäftigten sich mit bereits didaktisierten Filmmaterialien bzw. professionellen Kritiken. Durchgehend sehr gute Evaluierungen seitens der Studierenden belegen den Erfolg dieses Unterrichtsansatzes.

\section{Spielfilme im Deutschunterricht - die studentische Perspektive}

Das Vermitteln und Erleben lebendiger deutscher Sprache (und Kultur) in authentischen kommunikativen Situationen mittels Spielfilmen stellt für die überwiegende Mehrheit der Deutschlehrenden und -lernenden in Malaysia (immer noch) einen neuen Lehr- und Lernansatz dar. Nur wenige - z.B. Schüler auf internationalen Schulen - kamen jemals in den Genuss dieser „magischen Momente“, durch die das traditionell lehrbuch- und prüfungszentrierte Unterrichtsgeschehen an Schulen, Colleges, Sprachinstituten und Universitäten des Landes zweifelsohne bereichert werden würde. (SCHAAR; LAPASAU; OGASA 2013) Eine elektronische Umfrage aus dem Jahr 2009 unter 870 malaysischen Studierenden an vier Universitäten kam bereits zu dem Ergebnis, dass der Konsum von Fernsehsendungen, Musik- und Filmclips, Videos und Spielfilmen die bevorzugte Freizeitaktivität jugendlicher Malaysier ist und daher auch ein wesentlicher Bestandteil der Unterrichtsaktivitäten im Fremdsprachenunterricht sein sollte (SCHAAR; LAPASAU; NG 2013).

70 malaysische Deutschlernende vom INTEC und GMI (A-Level-German-Programme) und 98 BA-German Studierende der UPM (n=168) gaben ihre Zustimmung zur Teilnahme an einer empirischen Studie, welche die Erwartungen an deutschsprachige Spielfilme, den Medienkonsum allgemein, die Filmsehgewohnheiten und die Genrepräferenzen, die für die 
Schaar, T.; Chang, S.W.; Gilgen, A. - "Magische Momente" im Klassenraum?

Auswahl geeigneter Filme für den Unterricht eine wichtige Rolle spielen, näher untersuchte. Aus der Gesamtheit der gezeigten Spielfilme wurde ein Top 20-Ranking (aus Sicht der Studierenden) erstellt, das Lehrkräften die Auswahl von deutschen Spielfilmen für den Einsatz im DaF-Unterricht erleichtern soll.

\section{TABELLE 7}

Teilnehmer(innen) an der Studie

\begin{tabular}{llcll} 
Teilnehmer(innen) & Alter & männlich & weiblich & gesamt \\
\hline INTEC / GMI & $17-18$ & $48(68.5 \%)$ & $22(31.5 \%)$ & $70(100 \%)$ \\
UPM & $19-23$ & $8(8 \%)$ & $90(92 \%)$ & $98(100 \%)$ \\
gesamt & & $56(33.3 \%)$ & $112(66.6 \%)$ & $\mathbf{1 6 8}(100 \%)$
\end{tabular}

Die A-Level-German-Programme bereiten ausgewählte junge Malaysierinnen und Malaysier auf ein ingenieurswissenschaftliches Studium an deutschen Fachhochschulen sowie auf das dazu erforderliche Bestehen der DSH- bzw. TestDaF-Prüfung (B2, C1/GER) vor. Männliche Studierende überwiegen in diesen naturwissenschaftlich-technischen Studienprogrammen, während junge Frauen den BA-German Studiengang an der UPM klar dominieren. Im traditionell konservativen Malaysia gilt das Studium von Sprachen eher als „unmännlich“.

Die Teilnehmenden wurden während des Deutschunterrichts (UPM, INTEC) bzw. während thematisch spezifischer interkultureller Workshops (GMI) gebeten, in zwei Fragebögen (auf Deutsch mit englischer Übersetzung) die oben genannten Themenbereiche durch Anwendung der Fünf-Punkte-Likert-Skala in 6 geschlossenen Fragen positiv, neutral oder negativ zu bewerten. Die Antwortoptionen (absteigend) waren: 5 (I stimme voll zu), 4 (Ich stimme zu), 3 (unentschieden), 2 (Ich stimme nicht zu), 1 (I stimme gar nicht zu). Während der persönlichen Befragung erhielten ausgesuchte Teilnehmende die Möglichkeit ihre Stellungnahmen zu verschiedenen Aussagen zu begründen. In einem Pretest mit dem Semester 3 des BA-German Programms (UPM) wurden die Fragebögen getestet und geringfügige Veränderungen vorgenommen. Die Darlegung der Ergebnisse folgt den Fragestellungen der Studie.

Fragebogen 1

- Welches Medium - neben dem Lehrbuch - bevorzugen Sie, um eine Fremdsprache zu erlernen bzw. um ihre Kenntnisse zu vertiefen und zu erweitern?

- Welche Sendungen sehen Sie am liebsten? 
Schaar, T.; Chang, S.W.; Gilgen, A. - "Magische Momente" im Klassenraum?

- Aus welchen Ländern / Regionen kommen Ihre Lieblingsfilme /-serien?

- Welche Genres / Subgenres bevorzugen Sie?

- Was ist für Sie in einem Spielfilm besonders wichtig?

- Welche Erwartungen haben Sie speziell an deutsche Spielfilme?

Fragebogen 2

- Wie bewerten Sie die gesehenen Spielfilme? (10 Kriterien) (zusätzlich wurden die Teilnehmenden gebeten allgemeine / detaillierte Fragen zum Film zu beantworten)

Die Datenauswertung verzichtete sowohl auf Aussagen und Schlussfolgerungen basierend auf der sozialen Herkunft der Befragten und ihrer ethnischen Zugehörigkeit (Malaien, chinesische und indische Malaysier, Angehörige indigener Völker), da diese für die Studie irrelevant waren, als auch auf eine genderspezifische Analyse. So zeigten z.B. Genrepräferenzen, Sehgewohnheiten, die Herkunft der bevorzugten Spielfilme und Erwartungen an fiktionale Filmproduktionen entweder identische Resultate oder nur unwesentliche Abweichungen.

Um bereits erworbene Sprachkenntnisse zu vertiefen und zu erweitern, bevorzugen INTEC/GMI-Studierende - neben dem Lehrbuch - Songtexte (80,09\%), Filme / Videoclips $(70,8 \%)$ sowie Sprachlern-Apps wie Duolingo, Busuu u.a (56,02\%). Etwa die Hälfte der Collegestudierenden (49\%) zieht einen begleitenden Sprachkurs z.B. am Goethe-Institut in Betracht, 39,8\% favorisieren online-Sprachkurse. Nur 32\% lesen fremdsprachige Artikel. UPM-Studierende zeigen eine klare Präferenz für Filme/Videoclips (80,03\%) und Songtexte $(73,8 \%)$, vor Sprachlern-Apps (36,1\%), online-Kursen (26,3\%) und zusätzlichem Unterricht an Sprachinstituten (24,6\%). Lediglich 8,3\% machen sich die Mühe Artikel in der Fremdsprache zu lesen (Ho 2016).

Männliche und weibliche junge Malaysier sehen am liebsten Spielfilme (4.8), Fernsehserien/Soaps (4.3), Reality Shows (4.2) Cartoons (4.1) und Musikvideos (4.1). Koch-, Game- und Talkshows erfreuen sich noch einer gewissen Beliebtheit, während religiöse Programme (3.3), Dokumentationen (3.2), Nachrichtensendungen (3.1) und Sportübertragungen - mit Ausnahme von Fußball und Badminton - (2.9) eher selten angeschaut werden.

Hinsichtlich der Genrepräferenzen ist festzustellen, dass die überwiegend männlichen Deutschlernenden am INTEC/GMI Action- (4.4) und Science Fiction-Filme (4.35) sowie Animationsfilme (4.23), Cartoon Adaptionen (4.16), Komödien (3.95), Thriller (3.92) und Fantasyfilme (3.86) bevorzugen. Studierende an der UPM (überwiegend weiblich) zeigen eine 
Schaar, T.; Chang, S.W.; Gilgen, A. - „Magische Momente“ im Klassenraum?

Vorliebe für Komödien (4.55), Animations- (4.31), Action- (4.28) und Fantasyfilme (4.26), für Cartoon Adaptionen (4.11), Beziehungs-/Familiendramen (4.02), Thriller (4.02), Romanzen (3.92) sowie für Musicals (3.80). Ein gewisses Interesse besteht auch an Tierfilmen (3.83), Martial Arts Filmen (3.79), Horror-/Geisterfilmen (3.72), Coming-of-Age- (3.70) und Sozialdramen (3.63) sowie an Katastrophenfilmen (3.62). Einig sind sich alle Befragten in der Ablehnung von Kinder- (3.28), Kriegs- (3.25) und Sportfilmen (3.03) als auch von Spielfilmen, in denen politische Ereignisse bzw. Gerichtsverhandlungen thematisiert werden (3.0). Viele der Befragten favorisieren Spielfilme, die auf wahren Ereignissen basieren (4.49).

„Gute Filme“ - in der Wahrnehmung junger Malaysier(innen) - erzählen ,inspirierende Geschichten“ (4.82), ,vermitteln moralische Werte“ (4.69), enthalten, ,interessante Charaktere“ (4.59) „überraschende Wendungen und ein unerwartetes Ende“ (4.55). Sie zeigen „große Gefühle“ (4.37), haben einen „hohen Unterhaltungswert“ (4.31), und eine „besondere Atmosphäre“ (4.40). Sie faszinieren die Zuschauer mit „wunderschöner Filmmusik“ $(4,33)$, einer ,ansprechenden Ausstattung“ (4.22), einer „plot-getriebenen Handlung“ (4.22) und mit „realistisch wirkenden Spezialeffekten“ (4.18). „Berühmte Schauspieler(innen)“ (3.7), ein „happy-end“ (3.8), „Kameraarbeit, Dramaturgie, Licht, Ton, Schnitt“ (3.6) und die Tatsache, dass der Film von einer/m ,,bekannten Regisseur/in“ gedreht wurde, spielen bei der Beurteilung eines Filmes keine wesentliche Rolle.

Die Filmsehgewohnheiten der malaysischen Studierenden sowie ihre Vorstellung von „guten Filmen“ wurden und werden vor allem durch den Konsum von US-amerikanischen aber auch ostasiatischen Kino- und Fernsehfilmen sowie Serien geprägt. Sowohl die männlichen als auch die weiblichen Deutschlernenden bevorzugten Hollywood-Filme (4.3) vor Produktionen aus Korea / Japan (4.1) und chinesisch-sprachigen Spielfilmen aus Taiwan / Hong Kong / China (3.5). Indische Bollywood- bzw. tamilische Produktionen sowie Filme aus Malaysia selbst gehören zu den am wenigsten favorisierten (3.2 / 3.1). Europäische Filme, einschließlich deutscher Filme, waren den Befragten vor dem Einsatz im Unterricht fast völlig unbekannt, da diese in Malaysia kaum beworben werden und nur in ausgewählten Pay-TV-Kanälen (Astro) zur Verfügung stehen bzw. auf speziellen Filmfestivals (EU Film Festival) - die von jungen Zuschauern aber eher selten frequentiert werden - zu sehen sind. Somit kommt den DaFLehrkräften nicht selten eine Pionierrolle bei der Implementierung von deutschen Spielfilmen zu, die durch eine geschickte Auswahl zu einer positiven Wahrnehmung der deutschsprachigen Filmproduktionen führen sollte. 
Schaar, T.; Chang, S.W.; Gilgen, A. - "Magische Momente" im Klassenraum?

Tabelle 8 zeigt, dass das Interesse der Deutschlernenden vor allem auf das Erleben authentischer Sprache in realen Situationen, auf den Alltag in deutschsprachigen Ländern und die zwischenmenschlichen Beziehungen sowie auf Städte, Regionen und Landschaften ausgerichtet ist. Zudem erwarten die jungen Menschen spannende und unterhaltsame Geschichten.

\section{TABELLE 8}

Erwartungen der Studierenden an deutsche Spielfilme

\begin{tabular}{|c|c|}
\hline $\begin{array}{l}\text { Welche Erwartungen haben Studierende an deutsche Filme im L3 } \\
\text { Deutschunterricht in Malaysia? } n=168\end{array}$ & $\begin{array}{c}\text { mean } \\
5.0\end{array}$ \\
\hline Ich möchte meinen Wortschatz trainieren und erweitern. & $\begin{array}{c}4.8 \\
\text { ich stimme voll zu }\end{array}$ \\
\hline Ich möchte das Hörverstehen trainieren und verbessern. & $\begin{array}{c}4.7 \\
\text { ich stimme voll zu }\end{array}$ \\
\hline Ich möchte die deutsche Sprache in realen Situationen erleben. & $\begin{array}{c}4.6 \\
\text { ich stimme voll zu }\end{array}$ \\
\hline Ich möchte deutsche Schauspielerinnen und Schauspieler kennenlernen. & $\begin{array}{c}3.4 \\
\text { unentschieden }\end{array}$ \\
\hline Ich möchte mehr über die deutsche Gesellschaft lernen. & $\begin{array}{c}4.2 \\
\text { ich stimme zu }\end{array}$ \\
\hline $\begin{array}{l}\text { Ich möchte mehr über den deutschen Alltag wissen (wie die Menschen } \\
\text { leben, über Beziehungen, Familie, Formen des Zusammenlebens, } \\
\text { Ausländer, Festivitäten, Freizeitverhalten etc.). }\end{array}$ & $\begin{array}{c}4.7 \\
\text { ich stimme voll zu }\end{array}$ \\
\hline Ich möchte etwas über deutsche Geschichte lernen. & $\begin{array}{c}4.0 \\
\text { ich stimme zu }\end{array}$ \\
\hline $\begin{array}{l}\text { Ich möchte Anregungen über mögliche Reiseziele in Deutschland } \\
\text { bekommen (Städte, Regionen, Landschaften, Sehenswürdigkeiten). }\end{array}$ & $\begin{array}{c}4.7 \\
\text { ich stimme voll zu }\end{array}$ \\
\hline $\begin{array}{l}\text { Ich möchte deutsche Technologie "in Action" erleben (Autos, Züge, } \\
\text { Maschinen etc.). }\end{array}$ & $\begin{array}{c}4.1 \\
\text { ich stimme zu }\end{array}$ \\
\hline Ich freue mich auf spannende Geschichten. & $\begin{array}{c}4.6 \\
\text { ich stimme voll zu }\end{array}$ \\
\hline Ich möchte generell meinen Horizont erweitern. & $\begin{array}{c}4.5 \\
\text { ich stimme voll zu }\end{array}$ \\
\hline $\begin{array}{l}\text { Ich möchte meine Kultur mit der Kultur Deutschlands / Österreichs / der } \\
\text { Schweiz vergleichen. }\end{array}$ & $\begin{array}{c}3.7 \\
\text { ich stimme zu } \\
\end{array}$ \\
\hline $\begin{array}{l}\text { Ich möchte Filme sehen, die sich von meinen bevorzugten Filmen (USA, } \\
\text { Korea, Japan, China, Hongkong, Taiwan) vielleicht unterscheiden. }\end{array}$ & $\begin{array}{c}4.2 \\
\text { ich stimme zu }\end{array}$ \\
\hline Ich möchte unterhalten werden. & $\begin{array}{c}3.7 \\
\text { ich stimme zu }\end{array}$ \\
\hline Ich erwarte nicht wirklich viel von deutschen Filmen. & $\begin{array}{c}2.2 \\
\text { ich stimme nicht zu }\end{array}$ \\
\hline
\end{tabular}


Schaar, T.; Chang, S.W.; Gilgen, A. - "Magische Momente" im Klassenraum?

Unter Berücksichtigung der genannten Filmauswahlkriterien, der ermittelten Genrepräferenzen der Studierenden, der Verfügbarkeit der Filme mit (deutschen / englischen) Untertiteln und der Eignung für junge Malaysier(innen) zeigte der Verfasser an der UPM, am INTEC und am GMI über einen Zeitraum von drei akademischen Jahren (2013-2016) und unter verschiedenen didaktisch-methodischen Zielstellungen (Sprach- und Kulturvermittlung, Erstellung und Erprobung eines Vier-Phasen-Unterrichtsmodells, Anfertigung von Bachelorarbeiten, interkulturelle Workshops) 32 deutsche Spielfilme verschiedener Genres / Subgenres: Komödien, Coming-of-Age Dramen, interkulturelle Beziehungskomödien/ -dramen, Horrorund Actionfilme, Familiendramen sowie Literaturadaptionen und Geschichtsfilme. Zusätzlich zur Bearbeitung der jeweilig spezifischen Aufgabenstellung, wurden die Betrachter gebeten, den Spielfilm unter folgenden Kriterien zu beurteilen: der Unterhaltungswert, die schauspielerische Leistung, die Relevanz der erzählten Geschichte, das Ende, das Potential für intellektuelle Stimulanz, die Vermittlung von Aspekten der deutschen Kultur / Gesellschaft / Geschichte, Identifikationsmöglichkeiten mit Charakteren, die Möglichkeiten des Spracherwerbs, das Geeignet sein für junge Malaysier(innen).

Das aus diesen Umfragen erstellte Top 20-Ranking deutscher Spielfilme zeigt sehr deutlich, dass malaysische Studierende eine Vorliebe für eher „leichte“ und „klamaukige“ Unterhaltung, u.a. für Komödien von Til Schweiger, Matthias Schweighöfer und Bora Dagtekin zeigen.

TABELLE 9

Top 20-Ranking deutscher Spielfilme

Ranking Filmtitel (English Film Title), Erscheinungsjahr, Regisseur(e) (Zahl der Zuschauer) Bewertung von 5

\begin{tabular}{lll}
\hline 1 & Kokowääh, 2011, Til Schweiger & (49) 4.7 \\
\hline 2 & Barfuss (Barefoot), 2005, Til Schweiger & (55) 4.45 \\
\hline 3 & $\begin{array}{l}\text { Renn, wenn du kannst (Run, when you can), 2010, } \\
\text { Dietrich Brüggemann }\end{array}$ & (48) 4.4 \\
\hline 4 & Knockin on Heaven's Door, 1997, Thomas Jahn & (55) 4.35 \\
\hline 5 & Who am I, 2014, Baran bo Odar & (55) 4.3 \\
\hline 6 & Jenseits der Stille (Beyond Silence), 1996, Caroline Link & (54) 4.15 \\
\hline 7 & Keinohrhasen (Rabbit without Ears), 2007, Til Schweiger & (47) 4.05 \\
\hline 8 & Fack Ju Göthe (Suck me Shakespeer), 2013, Bora Dagtekin & (53) 4.0 \\
\hline 9 & $\begin{array}{l}\text { Die Nibelungen. Der Fluch des Drachen (The Curse of the } \\
\text { Dragon), 2004, Uli Edel }\end{array}$ & (45) 3.95 \\
\hline 10 & Die Fremde (When We Leave), 2010, Feo Aladag & (53) 3.9
\end{tabular}


Schaar, T.; Chang, S.W.; Gilgen, A. - "Magische Momente" im Klassenraum?

11 Schlussmacher (Break up Man), 2013, Matthias Schweighöfer, (62) 3.85 Torsten Künstler

\begin{tabular}{lll}
\hline 12 & Kebab Connection, 2004, Anno Saul & (53) 3.8 \\
\hline 13 & $\begin{array}{l}\text { Almanya - Willkommen in Deutschland (Welcome to } \\
\text { Germany), 2011, Yasemin Samdereli }\end{array}$ & (48) 3.7 \\
\hline 14 & Lola rennt (Run, Lola, Run), 1998, Tom Tykwer & (55) 3.65 \\
\hline 15 & Im Juli (In July), 2000, Fatih Akin & (53) 3.6 \\
\hline 16 & Same Same, But Different, 2009, Detlev Buck & (50) 3.45 \\
\hline 17 & Bella Martha (Mostly Martha), 2001, Sandra Nettelbeck & (54) 3.4 \\
\hline 19 & Eltern (Parents), 2013, Robert Thalheim & (56) 3.35 \\
\hline 20 & $\begin{array}{l}\text { Türkisch für Anfänger (Turkish for Beginners), 2012, } \\
\text { Bora Dagtekin }\end{array}$ & (52) 3.3 \\
\hline
\end{tabular}

Da die Mehrheit der Deutsch-Lernenden in Malaysia Muslime sind und die Integration von Muslimen in die deutsche Gesellschaft ein aktuelles Thema von politischer Relevanz und Brisanz ist, wurden auch die interkulturellen Beziehungskomödien /-dramen, vor allem jene, die sich auf Migrant(inn)en mit islamischem Hintergrund in Deutschland konzentrieren (Die Fremde, Kebab Connection, Almanya - Willkommen in Deutschland, Türkisch für Anfänger), interessiert verfolgt und überwiegend positiv wahrgenommen. Die Studierenden lernten so die in der deutschen Filmindustrie erfolgreichen deutsch-türkischen Schauspieler(innen) und Regisseure wie Fatih Akin, Bora Dagtekin, Sibel Kekilli, Elyas M`Barek sowie die Tatsache kennen, dass (deutsche) Türken die größte ethnische Minderheit in Deutschland bilden und die türkische Kultur in den größeren Städten des Landes wie Berlin, Köln, Frankfurt/Main, Stuttgart deutlich sichtbar ist. Die genannten Filme trugen auch dazu bei, muslimischen Stipendiaten, die sich am INTEC und GMI auf ihr Studium in Deutschland vorbereiten, „Sorgen“ vor eingeschränkten Möglichkeiten für ihre freie Religionsausübung zu nehmen.

Seit der Einführung des Final Year Research Projects im Jahr 2014 an der UPM wurden in BA-Abschlussarbeiten auch filmspezifische Themen bearbeitet - u.a. die Wahrnehmung (fremd)kultureller Aspekte insbesondere von Beziehungen in Til-Schweiger- und MatthiasSchweighöfer-Komödien (Kokowääh, Keinohrhasen, Schlussmacher, What a Man); die Wahrnehmung von „Ehrenmorden“ durch malaysische Studierende am Beispiel des Films Die Fremde und die Darstellung von „Ausländern“ in Fatih Akins Filmen (Im Juli, Gegen die Wand, Auf der anderen Seite, Soul Kitchen). Es wurden zudem Mütter-Töchter-Beziehungen, interkulturelle Beziehungen, Kinder-Eltern-Beziehungen sowie das Konzept Freundschaft in deutschen Filmen analysiert (Türkisch für Anfänger, Eltern, Same Same but Different) und 
Schaar, T.; Chang, S.W.; Gilgen, A. - "Magische Momente" im Klassenraum?

interkulturell mit der Situation in Malaysia verglichen. Die entsprechenden deutsche Spielfilme wurden aus dem Top-20 Ranking ausgewählt und verschiedenen Lernergruppen (Semester 3/A2, Semester 5/B1) gezeigt. Im Anschluss an die Vorführungen füllten die Teilnehmenden anonym standardisierte Fragebögen aus, die u.a. darauf abzielten, die Szenen des Films herauszufiltern, die aus Perspektive der Lernenden auffällige, für sie „fremde“ Aspekte der deutschen Kultur gezeigt hätten. Anschließend wurde eine Diskussion angeregt, in der die Studierenden nochmals anmerken konnten, was ihnen aufgefallen war, wobei nun die Gelegenheit bestand, nachzufragen, Fragen aufzugreifen und zu klären (AZAM 2014; CHONG 2016; Chua 2014; Ho 2016; LaU 2015; Lee 2015; Lim 2014;, Sharon Lai 2014).

Aus diesen BA-Abschlussarbeiten, aus Unterrichtsgesprächen und Essays lässt sich erkennen, dass junge malaysische Deutschlernende in der konstruierten fiktiven Realität der Spielfilme als besonders positiv empfinden, dass Menschen in Deutschland offen ihre Gefühle ausdrücken und darüber sprechen würden, dass sie ehrlich kritisch und respektvoll miteinander umgehen, ihre Meinungen vertreten und über konträre Standpunkte diskutieren. Pünktlichkeit werde als Respekt und Achtung vor dem Anderen verstanden, Freunde hätten eine große Bedeutung, manchmal sogar eine größere Bedeutung als Familienmitglieder. Deutsche organisierten, planten und setzten sich Ziele für die Zukunft. Das Lehrer-Schüler-Verhältnis sei im Vergleich zu Malaysia deutlich entspannter; im Unterricht werde eine Diskussionskultur gefördert (CHUA 2014; Ho 2016; LAU 2015; LEE 2015; LIM 2014).

Der preisgekrönte Film Die Fremde (2010, Feo Aladag), der sich mit der grausamen Praxis der sogenannten „Ehrenmorde“ am Beispiel einer in Berlin lebenden türkischen Familie beschäftigt, wurde als ein „sehr wichtiger Film“ (Nr. 10), wahrgenommen. 77\% der 53 Betrachter stimmten allerdings zu, dass die Protagonistin Umay durch das Verlassen ihres türkischen Ehemannes und die spätere Liebesbeziehung zu einem Deutschen „Schande über ihre Familie“ gebracht hatte. (Eine Studentin verwies hier auf die malaiische Redensart „Den Himmel findet die Frau unter den Füßen des Ehemannes“) Die angeordnete Ermordung Umays zur Wiederherstellung der Familienehre durch den Bruder lehnten jedoch alle als „unmenschlich“ ab. 19\% kritisierten den Film als anti-islamisch. Der Film sei zu grausam, die Handlung habe nichts mit der Lebenswirklichkeit in Malaysia zu tun, es werde zu viel Türkisch gesprochen, er verherrliche „sündiges Leben“, zeige nicht den „wahren Islam“, sondern nur „die türkische Auslegung“. Außerdem entwickele sich die Geschichte sehr langsam und zeige keine deutsche Kultur (AZAM 2014). 
Schaar, T.; Chang, S.W.; Gilgen, A. - „Magische Momente“ im Klassenraum?

Der thematisch komplexe, auf wahren Ereignissen basierende Film Same, same, but different (2009, Detlev Buck), in dem sich der junge Deutsche, Benjamin Prüfer, in die HIVpositive kambodschanische Prostituierte, Sreykeo Solvan, verliebt und diese trotz der Ansteckungsgefahr auch heiratet, galt für die Lernenden zwar als „,nicht sehr relevant“ (Nr.16), sie diskutierten diesen dennoch leidenschaftlich und kontrovers. Während interkulturelle Beziehungen - aus religiösen und juristischen Gründen - von offizieller staatlicher Seite in Malaysia eher abgelehnt werden (Attorney General of Malaysia, Abdul Gani Patail, Rakyattimes, 19. November 2014), sprach sich die überwiegende Mehrheit der Student(inn)en dafür aus. Obgleich Herausforderungen hinsichtlich der gemeinsamen Sprache (3.7), der Religion (3.3), der Erziehung der Kinder (3.3), unterschiedlicher Wertvorstellungen z.B. über Familie, Liebe, Tabus, Interessen und Hobbies (3.1) erkannt und benannt wurden, glauben die jungen Menschen, dass Liebe, Toleranz und Kompromisse zur Lösung dieser Probleme führen würden. Eine interkulturelle Beziehung könnten sie sich am ehesten mit Partnern aus Ost- und Südostasien (Japan, Korea, Thailand - 4.1), Europa (3.7) und Nordamerika (2.7) vorstellen (SHARON LAI 2014).

Häufig „fremdelten“ junge Malaysierinnen und Malaysier mit visualisierten antiautoritären Erziehungskonzepten, respektlosen, unhöflichen Kindercharakteren (Eltern) mit Patchwork-Familien, mit dem Zusammenleben verschiedengeschlechtlicher Freunde in derselben Wohnung und mit allzu selbstbewusst auftretenden, unangepassten und sexuell offenen weiblichen Figuren (insbesondere Muslimas - Türkisch für Anfänger, Die Fremde, Fack Ju Göthe). Sie übten Kritik an der hohen Scheidungsrate in Deutschland, an der häufigen Benutzung von Schimpfwörtern sowie am „ständigen Alkoholgenuss“ (im Film) und lehnten die Akzeptanz der offenen gelebten Homosexualität als normal, außerehelichen Sex und die verbale Konfrontation von Partnern in einer Beziehung strikt ab (CHUA 2014; Ho 2016; LAU 2015; LEE 2015; LIM 2014).

Im Rahmen eines Forschungsprojekts (2014-2016) entwickelten Ogasa und Schaar ein auf den Ergebnissen der BA-Abschlussarbeiten und auf theoretischen Überlegungen zu Möglichkeiten des Erwerbs interkultureller Kompetenz basierendes Unterrichtsmodell, welches die Lernenden durch den Einsatz von ausgewählten, didaktisierten Filmsequenzen für fremd- und eigenkulturelle gesellschaftliche und soziale Aspekte sensibilisierte. Das Modell basiert auf vier Phasen, welche die Lernenden, angeleitet durch die Lehrperson, und mit Hilfe des Einsatzes einer Filmsequenz durchlaufen haben: 1) Beobachtung, 2) Wahrnehmung und Deutung, 3) Reflexion und Bewusstwerdung der eigenen Kultur, 4) Re-Interpretation. Erste 
Schaar, T.; Chang, S.W.; Gilgen, A. - „Magische Momente" im Klassenraum?

Auswertungen der Studie zeigten, dass der methodische Einsatz von Spielfilmen /

Filmsequenzen zur interkulturellen Sensibilisierung sowie das erstellte Unterrichtsmaterial (didaktisierte Arbeitsblätter) nicht nur sehr positiv von den Lernenden aufgenommen wurde, sondern, dass sich alle Befragten für mehr Spielfilme im Unterricht Deutsch als Fremdsprache in Malaysia ausgesprochen haben (OGASA; SCHAAR 2017).

\section{Literaturverzeichnis}

ACHARYA, Swati. Arbeit mit Spielfilmen im Unterricht Deutsch als Fremdsprache: Didaktisierungsmöglichkeiten des Films Sonnenallee. In: WELKE, Tina; FAISTAUER, Renate (Org.). Lust auf Film heißt Lust auf Lernen. Der Einsatz des Mediums Film im Unterricht Deutsch als Fremdsprache. Wien: Praesens, 2010. p. 151-165.

AuswärTigeS AMT. Deutsch als Fremdsprache weltweit. Datenerhebung 2015. Berlin, 2015.

AZAM, Fatin Fatehah bt Hasnal. Malaysian Students' Perception of the German Movie "Die Fremde" by Feo Aladag. BA Thesis. FBMK/JBA/UPM, Serdang, 2014.

BIECHELE, Barbara. "Ich sehe was, was du nicht siehst" - Reflexionen zum Lernen mit Spielfilmen im Unterricht Deutsch als Fremd- oder Zweitsprache. In: ESSER, Ruth; KRUMM, Hans-Jürgen (Org.). Bausteine für Babylon: Sprache, Kultur, Unterricht. Festschrift zum 60. Geburtstag von Hans Barkowski. München: iudicum, 2007. p. 194-205.

BIECHELE, Barbara. Film/Video/DVD in Deutsch als Fremdsprache. In: BARKOWSKI, Hans; WolfF, Armin. Umbrüche. Materialien Deutsch als Fremdsprache 76. Regensburg: FaDaF, 2006. p. 310-328.

BIECHELE, Barbara. Verstehen braucht Sehen: entdeckendes Lernen mit Spielfilm im Unterricht Deutsch als Fremdsprache. In: WelKE, Tina; FAISTAUER, Renate (Org.). Lust auf Film heißt Lust auf Lernen. Der Einsatz des Mediums Film im Unterricht Deutsch als Fremdsprache. Wien: Praesens, 2010. p. 13-32.

BRANDI, Marie-Luise. Video im Deutschunterricht. Eine Übungstypologie zur Arbeit mit fiktionalen und dokumentarischen Filmsequenzen. Fernstudieneinheit 13. Berlin: Langenscheidt, 1996.

CHONG, Chan Yee. Historical Feature Film as an Educational Tool in Teaching History of Germany to BA German Students at Universiti Putra Malaysia. BA Thesis. FBMK/JBA/UPM, Serdang, 2016.

CHUA, Wan Suet. Students' Perception and Comparisons of Cultural Aspects in Germany and Malaysia based the films SCHLUSSMACHER and WHAT A MAN by MATTHIAS SCHWEIGHÖFER. BA Thesis. FBMK/JBA/UPM, Serdang, 2014.

CHUDAK, Sebastian. Der deutsche Film auf Erfolgskurs! Warum nicht auch im Deutsch-alsFremdsprache Unterricht? Fremdsprache Deutsch. Heft 36 "Sehen(d) lernen”, p. 14-16, 2007.

CHUDAK, Sebastian. Einsatz der Textsorte "Film" im Fremdsprachenunterricht. Überlegungen zur Stellung von audiovisuellen Unterrichtsmitteln in neuen Lehrwerken für DaF. Studia Germanica Posnaniensia XXXI. Wydawnictwo Naukowe. Poznan, p. 113-129, 2008.

CHUDAK, Sebastian. Lehrwerk ... oder vielleicht doch Filme? Überlegungen zu den Möglichkeiten effektiver Förderung der interkulturellen kommunikativen Kompetenz im Unterricht DaF durch den Einsatz von Lehrwerken und Filmen. In: WelKe, Tina; FAISTAUER, Renate (Org.). Lust auf Film heißt Lust auf Lernen. Der Einsatz des Mediums Film im Unterricht Deutsch als Fremdsprache. Wien: Praesens, 2010. p. 61-84. 
Schaar, T.; Chang, S.W.; Gilgen, A. - "Magische Momente" im Klassenraum?

ETYA, Lee. Der Einsatz von Spielfilmen Unterricht Deutsch als Fremdsprache am Beispiel Das fliegende Klassenzimmer. In: WELKE, Tina; FAISTAUER, Renate (Org.). Lust auf Film heißt Lust auf Lernen. Der Einsatz des Mediums Film im Unterricht Deutsch als Fremdsprache. Wien: Praesens, 2010. p. 166-185.

FAISTAUER, Renate. Prinzipien im Sprachunterricht $=$ Prinzipien für die Arbeit mit Filmen im Sprachunterricht?! In: WelKe, Tina; FAISTAUER, Renate (Org.). Lust auf Film heißt Lust auf Lernen. Der Einsatz des Mediums Film im Unterricht Deutsch als Fremdsprache. Wien: Praesens, 2010. p. 33-45.

GAMKRELIDZE, Dali. Das Versprechen (Margarete v. Trotta) - ein Didaktisierungsvorschlag. In: WelKe, Tina; FAISTAUER, Renate (Org.). Lust auf Film heißt Lust auf Lernen. Der Einsatz des Mediums Film im Unterricht Deutsch als Fremdsprache. Wien: Praesens, 2010. p. 186-193.

GÜGOLD, Barbara. Zum Einsatz des Spielfilms im Fremdsprachenunterricht. Deutsch als Fremdsprache, 4, p. 238-241, 1991.

HARMS, Michael. Augen auf im Fremdsprachenunterricht - psychologische und didaktische Aspekte des Lernens mit Bildmedien. In: DuXA, Susanne; Hu, Adelheid; ScHMENK, Barbara (Org.). Grenzen überschreiten. Menschen, Sprachen, Kulturen. Festschrift für Inge C. Schwerdtfeger. Tübingen: Narr, 2005. p. 245-256.

Ho, Shu Yun. German Feature Films as Educational Tool to create Cultural Awareness for Bachelor of German Language Students at Universiti Putra Malaysia. BA Thesis. FBMK/JBA/UPM, Serdang, 2016.

KÄRCHNER-OBER, Renate. The German Language is Completely Different from the English Language. Besonderheiten des Erwerbs von Deutsch als Tertiärsprache nach Englisch und einer NichtIndogermanischen Erstsprache. Tübingen: Stauffenberg, 2009.

KING, Jane. Using DVD feature films in the EFL classroom. The weekly column. Article 88, p. 509-5, fev. 2002.

LAU. Kah Lin. Young People in Germany and Malaysia - a Comparison. BA Thesis. FBMK/JBA/UPM, Serdang, 2015.

LAY, Tristan. Film und Video im Fremdsprachenunterricht. Eine empirisch quantitative Erhebung zur didaktisch-methodischen Implementierung filmspezifischer Arbeit im universitären Deutschstudium Taiwans. Zeitschrift für Interkulturellen Fremdsprachenunterricht, v. 14, n. 1, p. 107-153, 2009.

LEE, Sock Peng. Perception of the German family in feature films by Malaysian Students. BA Thesis. FBMK/JBA/UPM, Serdang, 2015.

LIM. Sin Yee. The Depiction of Family and Relationships in selected Comedies (KOKOWÄÄH and KOKOWÄ̈̈H 2) by TIL SCHWEIGER. BA Thesis. FBMK/JBA/UPM, Serdang, 2014.

Ministry OF Higher EdUCATION. Future Direction of Language Education in Malaysia. Putrajaya, 2010.

OGASA, Nicole; SCHAAR, Torsten. Handeln zwischen den Kulturen will gelernt sein. Vorstellung eines Unterrichtsmodells zur Entwicklung interkultureller Kompetenz im Fremdsprachenunterricht. In: AKKRAMAS, Pakini; FunK, Hermann; TraORE, Salifou (Org.). Deutsch als Fremdsprache im Spannungsfeld zwischen Globalisierung und Regionalisierung. Peter Lang, 2017. p. 215228.

PRASAD, N.V.; SHANTHI, Balraj. Developing Media Literacy Practice among Secondary School Students in Malaysia: Case Studies of Video Making on Environmental Issues. Media Watch, v. 4, n. 1, p. 95-105, 2013.

Prasad, N.V.; RaO, Aaron; Dollah, Mohd. Zain. Young People and creative Video Production: A Case Study of Media Making on Issues of Stress and Friendship among Secondary School Students in Malaysia. Journal of Arts, Science \& Commerce, v. III, n. 4, out. 2012, p. 83-89. 
Schaar, T.; Chang, S.W.; Gilgen, A. - "Magische Momente" im Klassenraum?

RAABE, Horst. "Das Auge hört mit". Sehstrategien im Fremdsprachenunterricht. In: RAMPILLON, Ute; ZimmermanN, Guenter (Org.). Strategien und Techniken beim Erwerb fremder Sprachen. Ismaning: Hueber, 1997. p. 150-172.

SASS, Anne. Filme im Unterricht - Sehen(d) lernen. Fremdsprache Deutsch. Heft 36 "Sehen(d) lernen", p. 5-13, 2007.

SCHAAR, Torsten; LAPASAU, Merry; OGASA, Nicole. Teaching German as a Third language using DVD Feature Films in Malaysia. Studies on Foreign Languages and Cultures, v. 2, Serdang, UPM Press, p. 95-113, 2013.

SCHAAR, Torsten; LAPASAU, Merry; NG, Shwee Fang. Reading Habits of Malaysian students beyond Classrooms. Studies on Foreign Languages and Cultures, v. 2, Serdang, UPM Press, p. 114138, 2013.

SCHAAR, Torsten et al. Bachelor's Degree in German Studies and Then? The Professional Development of the 2008 BA German Graduates from Universiti Putra Malaysia. Global Journal of Business and Social Science Review, v. 2, n. 1, p. 499-509, 2015.

SCHAAR, Torsten. Career Perspectives of BA German Graduates In Malaysia: A Case Study Of The 2009-2011 Graduates From Universiti Putra Malaysia. IOSR Journal Of Humanities And Social Science (IOSR-JHSS), v. 21, n. 10, ver. 10, p. 28-40, out. 2016.

SCHAAR, Torsten; OGASA, Nicole. Was kommt nach dem Deutschstudium? Zu beruflichen Perspektiven der Absolvent(inn)en des BA German-Programms an der Universiti Putra Malaysia. In: AKKramas, Pakini; FunK, Hermann; Traore, Salifou (Org.). Deutsch als Fremdsprache im Spannungsfeld zwischen Globalisierung und Regionalisierung. Peter Lang, 2017. p. 47-64.

SCHWERDTFEGER, Inge Christine. Sehen und Verstehen. Zur Arbeit mit Film und Video im Fremdsprachenunterricht. Berlin: Langenscheidt, 1989.

SCHWERDTFEGER, Inge Christine. Übungen zum Hör-Sehverstehen. In: BRAUSCH, Kar-Richard; CHRIST, Herbert; KRUMM, Hans-Jürgen (Org.). Handbuch Fremdsprachenunterricht. Tübingen: A. Francke (UTB), 2003. p. 299-302.

SHANTHI, Balraj; KHOO, Seok Wah. Voices for Change: Media Literacy and a Case Study on Designing of Opportunities for Learning Internet Radio in Malaysia. Media Asia, v. 33, n. 1-2, p. 87-98, 2006.

SHARON LAI, Mei Lin. The Perception of Intercultural Relationships by Malaysian Students based on the Film "Same same but different". BA Thesis. FBMK/JBA/UPM, Serdang, 2014.

STORK, Matthias. Lights, Camera, Action. Zur Synthese von Film und Drama im interkulturellen Englischunterricht. Frankfurt/Main: Univ.-Bibliothek, 2012.

Thesen zur Arbeit mit Film im Unterricht Deutsch als Fremd- und Zweitsprache (DaF/Z). In: WeLKE, Tina; FAISTAUER, Renate (Org.). Lust auf Film heißt Lust auf Lernen. Der Einsatz des Mediums Film im Unterricht Deutsch als Fremdsprache. Wien: Praesens, 2010. p. 239-241.

TONSERN, Clemens. Der österreichische Spielfilm im Bereich DaF: warum es sich lohnt, das ewige Dornröschen wach zu küssen. In: WELKE, Tina; FAISTAUER, Renate (Org.). Lust auf Film hei $\beta t$ heißt Lust auf Lernen. Der Einsatz des Mediums Film im Unterricht Deutsch als Fremdsprache. Wien: Praesens, 2010. p. 211-228.

TsCHUDINOWA, Elena. Der Einsatz von Spielfilmen im studienbegleitenden Unterricht. In: WeLKE, Tina; FAISTAUER, Renate (Org.). Lust auf Film heißt Lust auf Lernen. Der Einsatz des Mediums Film im Unterricht Deutsch als Fremdsprache. Wien: Praesens, 2010. p. 194-210.

WELKE, Tina. Ein Plädoyer für die Arbeit mit Kurzfilmen. Fremdsprache Deutsch. Heft 36 "Sehen(d) lernen", p. 21-25, 2007.

WELKE, Tina. Stereotypen als Chance. Zur Arbeit mit Filmgenres. In: WELKE, Tina; FAISTAUER, Renate (Org.). Lust auf Film heißt Lust auf Lernen. Der Einsatz des Mediums Film im Unterricht Deutsch als Fremdsprache. Wien: Praesens, 2010. p. 111-132.

WelKe, Tina; FAISTAUER, Renate (Org.). Lust auf Film heißt Lust auf Lernen. Der Einsatz des Mediums Film im Unterricht Deutsch als Fremdsprache. Wien: Praesens, 2010.

Pandaemonium, São Paulo, v. 21, n. 35, set.-dez. 2018, p. 87-120 
Schaar, T.; Chang, S.W.; Gilgen, A. - "Magische Momente" im Klassenraum?

Recebido em 26 de janeiro de 2018 Aceito em 01 de maio de 2018

Pandaemonium, São Paulo, v. 21, n. 35, set.-dez. 2018, p. 87-120 